\title{
Patient Independent Representation of the Detailed Cardiac Ventricular Anatomy
}

\author{
Bruno Paun ${ }^{\mathrm{a}, *}$, Bart Bijnens ${ }^{\mathrm{a}, \mathrm{b}, \mathrm{c}}$, Tinen Iles ${ }^{\mathrm{d}}$, Paul A. Iaizzo ${ }^{\mathrm{d}}$, Constantine \\ Butakoffa \\ ${ }^{a}$ Universitat Pompeu Fabra, Department of Information and Communication \\ Technologies, c. Roc Boronat 138, 08018 Barcelona, Spain \\ ${ }^{b}$ ICREA, Passeig Lluís Companys 23, 08010 Barcelona, Spain \\ ${ }^{c}$ KU Leuven, Oude Markt 13, 3000 Leuven, Belgium \\ ${ }^{d}$ University of Minnesota, Visible Heart ${ }^{\circledR}$ Laboratory, Department of Surgery, \\ Minneapolis, Minnesota, USA
}

\begin{abstract}
Reparameterization of surfaces is a widely used tool in computer graphics known mostly from the remeshing algorithms. Recently, the surface reparameterization techniques started to gain popularity in the field of medical imaging, but mostly for convenient $2 \mathrm{D}$ visualization of the information initially represented on 3D surfaces (e.g. continuous bulls-eye plot). However, by consistently mapping the $3 \mathrm{D}$ information to the same $2 \mathrm{D}$ domain, surface reparameterization techniques allow us to put into correspondence anatomical shapes of inherently different geometry. In this paper, we propose a method for anatomical parameterization of cardiac ventricular anatomies that include myocardium, trabeculations, tendons and papillary muscles. The proposed method utilizes a quasi-conformal flattening of the myocardial surfaces of the left and right cardiac ventricles and extending it to cover the interior of the cavities using the local coordinates given by the solution of the Laplace's equation. Subsequently, we define a geometry independent representation for the detailed cardiac left and right ventricular anatomies that can be used for convenient visualization and statistical analysis of the trabeculations in a population. Lastly we show how it can be used for mapping the detailed cardiac anatomy between different hearts, which is of considerable
\end{abstract}

\footnotetext{
${ }^{*}$ Corresponding author. E-mail: bruno.paun@upf.edu. Postal address: Universitat Pompeu Fabra, Department of Information and Communication Technologies, c. Tànger 122 - 140, 08018 Barcelona, Spain
} 
interest for detailed cardiac computational models or shape atlases.

Keywords: parameterization, heart representation, cardiac coordinate system, surface flattening, cardiac trabeculations, conformal mapping

\section{Introduction}

Surface parameterization is a bijective mapping from a suitable parameter domain to the surface. As the parameter domain itself is usually a surface, the parameterization represents a mapping from one surface onto another, providing that the surfaces have the same topology (Sheffer et al., 2006; Floater and Hormann, 2005). In computational geometry the surfaces are approximated by 3D triangulated meshes and their parameterization is a process of creating piecewise linear mappings between the mesh and simpler triangulated domains such as planar regions, simplicial complexes or spheres (Sheffer et al., 2006). Surface parameterization is most frequently used in the field of computer generated imagery (CGI) as a method for mapping textures onto surfaces to add realism to the simplified polygonal meshes. There are also many applications in numerous problems such as texture synthesis, detail transfer, mesh completion, mesh editing, remeshing, mesh compression, object morphing and others.

Planar parameterization of general surfaces always introduces distortion in either angles or areas as stated by theorema egregium, and isometric (distance preserving or zero distortion) mappings are possible only for developable surfaces with zero Gaussian curvature. Thus, parameterization of arbitrary surfaces used in practice are mappings which minimize these distortions, such as conformal (angle preserving), equiareal or authalic (area preserving) and mappings which minimize some combinations of angle and area distortion. Planar mapping methods can be additionally divided into free and fixed boundary mappings. Fixed boundary mappings transform the boundary of a mesh to a predefined boundary in the $2 \mathrm{D}$ domain while boundary free methods compute the boundary of $2 \mathrm{D}$ domain as part of the solution that reduces the distortion.

Many of the planar parameterization methods are built upon Tutte's formulation of graph embedding, which directly applies to the meshes (Tutte, 1963). These parameterizations normally are performed in two stages. First, the boundary vertices of the 3D surface mesh are mapped to the boundary of the predefined $2 \mathrm{D}$ region. Afterwards, the positions of the remaining 
vertices are obtained by solving a system of linear equations (Pinkall and Polthier, 1993). Every mesh, which satisfies the Delaunay criterion, will have a bijective flattening map as suggested by Kharevych et al. (2006).

In medical imaging, harmonic mappings were first applied in neurology for the analysis and visualization of brain surface, where the complex brain structure was mapped to a sphere or a planar domain (Hurdal et al., 2000; Haker et al., 2000; Gu et al., 2004; Joshi et al., 2007; Wang et al., 2012). In cardiology, reparameterization is mostly used to visualize any functional information of the ventricular cavities on a discretized or continuous bull's-eye plot (Cerqueira et al., 2002). Such information can be coming from imaging modalities or electro-physiological measurements (Soto-Iglesias et al., 2013). A similar approach has recently been applied to the description of atria using a standardized unfold map or rectangular unfold map for the analysis of atrial fibrillation patients (Tobon-Gomez et al., 2015; Karim et al., 2014). Recently, harmonic spherical mapping was applied in hepatology for definition of an inter-patient liver coordinate system (Vera et al., 2014). Surface parameterization was also employed for creation of a normalized parametric domain for comparison of left ventricular function across subjects (Garcia-Barnes et al., 2010). Young et al. (2006) developed a finite element parameterization method for regional analysis of 3D myocardial function and construction of a 3D model of infarct geometry combined with 3D strain information, which was also used by Zhang et al. (2014) for mapping of left ventricular regional remodeling due to myocardial infarction. Another example of normalized domain is a tissue sampling map proposed by Young et al. (2011) used for coregistration of CMRI and ex-vivo tissue sampling.

When talking about detailed cardiac anatomy, it is necessary to mention that, although the current imaging techniques made screening of those structures feasible ex vivo, their accurate segmentation still represents a challenging task. M. Gao et al. recently proposed a method for a topologically accurate segmentation by restoring missing topological structures of initially given segmentations (Gao et al., 2013), which allowed them to preform morphological analysis of such structures in the left ventricle (LV) (Gao et al., 2014).

In this paper, we propose a method for a patient independent anatomical parameterization of the interior of cardiac ventricles for the representation of the detailed ventricular anatomy (myocardium, trabeculations, papillary muscles, etc.). The proposed method represents a generalization of the parameterization procedure proposed by Paun et al. (2015) used for pa- 


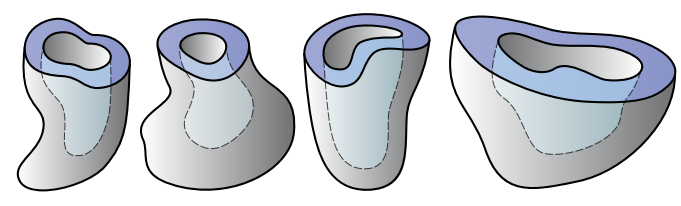

Figure 1: Examples of different shapes of thick shell structures.

rameterization of the detailed cardiac anatomy of left ventricles, which in general have a ellipsoidal-like shape. Unfortunately, that method introduces unwanted distortion and folding when applied to the more complex shapes such as the right ventricle (RV). To overcome such limitations we propose a general method that can be applied to objects of an arbitrary shape.

\section{Methods}

\subsection{Overview of the Geometry Independent Representation}

In this section we will introduce a general framework for representing any object $M$ with an overall shape of a thick hemispherical shell (in the following text we will call them shell-like objects for simplicity) based on the fact that it is homeomorphic to a cylinder. A number of examples of such objects can be seen in Fig. 1. In order to establish the homeomorphism, apart from the object $M$ itself, represented by either a surface or volumetric mesh, we will also require an enclosing surface $S$, usually represented by a triangulated mesh, that contains the whole $M$ in its interior.

The whole procedure of calculating the mapping can be described by the following steps (explained in the following sections and illustrated in Fig. 2):

1. Mapping of $S$ to a suitable planar domain $D$.

(a) Specifications of landmarks on $S$ to impose a consistent orientation.

(b) Conformal or quasi-conformal flattening.

2. Mapping of $M$ to the volumetric domain defined by the base $D$ (usually a cylinder or a prism).

For convenience, the most common symbols used throughout the paper are listed in Table 1. 


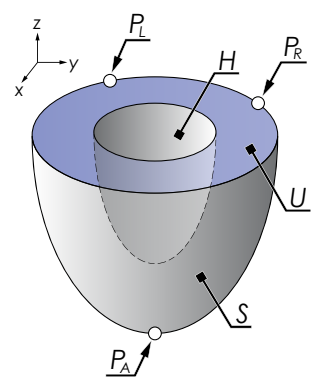

a)

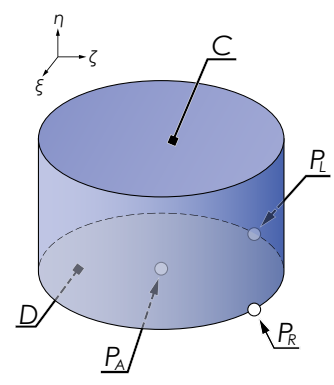

b)

Figure 2: Illustration of domain of interest with corresponding surfaces and landmark points. a) Domain $U$ defined by enclosing surface $S$, interior surface $H$, and landmark points $P_{A}, P_{L}$ and $P_{R}$. b) Cylinder domain $C$ of unit height defined by planar domain $D$ and corresponding landmarks. Symbols: $P_{L}$ - left boundary landmark; $P_{R}$ - right boundary landmark; $P_{A}$ - apical landmark; $S$ - enclosing surface; $H$ - interior surface; $U$ - domain between $S$ and $H ; D$ - planar domain; $C$ - cylinder.

\subsection{Specification of the Landmark Points on the Enclosing Surface}

In order to define a common representation for the shell-like objects and achieve a unique correspondence between different instances of such objects, we propose to fix three landmark points on $S$ (see Fig. 2). These points will be mapped to predefined locations in the planar domain, thus removing orientation ambiguity due to rotational and mirror symmetry. Two points, $P_{L}, P_{R}$, related to some easily identifiable features of the object, should be located on the boundary $\partial S$. The third point, $P_{A}$, should represent the position of the pole (apex) of the surface $S$.

\subsection{Mapping of the Enclosing Surface to the Planar Domain}

The identification of the landmarks on $S$ is followed by the mapping step of the surface $S$ to the planar domain. The planar domain represents the base of the corresponding normalized reference frame and can be represented by any convex planar shape. As implied by Riemann Mapping Theorem (Riemann and Wilkins, 1851), any surface homeomorphic to a disk can be conformally mapped into any simply-connected region of the plane. The method we use here is the same as the one used in the paper of De Craene et al. (2012).

A bijective mapping $\varphi: S \subset \mathbb{R}^{3} \longrightarrow D \subset \mathbb{R}^{2}$, where $S$ is the enclosing surface and $D$ is the corresponding planar domain, can be computed by 


\begin{tabular}{ll}
\hline \multicolumn{1}{c}{ Table of symbols } \\
\hline$S$ & enclosing surface \\
$H$ & interior surface \\
$U$ & domain between $S$ and $H$ \\
$\partial S$ & boundary of $S$ (curve) \\
$M$ & shell-like object \\
$D$ & surface $S$ mapped to the planar domain \\
$\partial D$ & boundary of $D$ (curve) \\
$N$ & object $M$ mapped to the cylinder \\
$P_{i}$ & a landmark (specified by the subscript) \\
\hline
\end{tabular}

Table 1: List of symbols used in the manuscript.

solving the following system of linear equations:

$$
\begin{gathered}
\left\{\begin{array}{c}
L_{S \backslash \partial S} \cdot X_{D \backslash \partial D}=0 \\
X_{\partial D}=X
\end{array}\right. \\
X=\left(\begin{array}{cc}
\cos \left(t_{0}\right) & \sin \left(t_{0}\right) \\
\cos \left(t_{1}\right) & \sin \left(t_{1}\right) \\
\vdots & \vdots \\
\cos \left(t_{B}\right) & \sin \left(t_{B}\right)
\end{array}\right)
\end{gathered}
$$

where $t_{i} \in[0,2 \pi]$ and $B$ is the number of vertices on $\partial S$.

The desired (fixed) boundary coordinates $\xi, \zeta$ are given by the columns of matrix $X . L_{S \backslash \partial S}$ represents the Laplacian matrix of the surface $S$ with the rows corresponding to its boundary $\partial S$ removed. $X_{\partial D}$ and $X_{D \backslash \partial D}$ are the $\xi, \zeta$ coordinates of the points on the planar domain corresponding to the boundary and the interior, respectively.

The above methodology provides a simple method for mapping $S$ to the planar domain $D$ where its corresponding boundary edge $\partial S$ is mapped to the perimeter of $D$. To impose a unique correspondence between different enclosing surfaces their landmarks $P_{L}, P_{R}$ and $P_{A}$ are mapped to the same predefined locations on the planar domain.

\subsection{Mapping of the Shell-like Objects to a Cylinder}

Let $\psi: M \rightarrow C$ be the bijective mapping of the object $M \subset \mathbb{R}^{3}$ onto a cylinder $C \subset \mathbb{R}^{3}$. Let $\psi\left(v_{M}\right)=v_{C} \in C$ for any $v_{M} \in M$. In this section we 
will provide the procedure to calculate the $\psi$ using the ideas of Yezzi and Prince (2003).

Let $S$ be the enclosing surface of the object $M, H$ the interior surface of $M, U=M \backslash(S \cup H)$ the domain between the $S$ and $H$ (see Fig. 2). Let $f(x, y, z)$ be a scalar field, the solution to the Laplace's equation in $U$ :

$$
\Delta f(x, y, z)=0
$$

with the following boundary conditions

$$
\begin{aligned}
& f(x, y, z)=-1, \forall(x, y, z) \in S \\
& f(x, y, z)=1, \forall(x, y, z) \in H
\end{aligned}
$$

Then for every point $v_{U} \in U$ we can find a unique integral curve $c(t)=$ $\left(c_{x}(t), c_{y}(t), c_{z}(t)\right)$ of the vector field $\nabla f$ such that $v_{U} \in c(t)$. The curve $c(t)$ can be calculated by solving the following system of equations:

$$
\frac{d c(t)}{d t}=\nabla f(c(t))
$$

with the condition

$$
c\left(t_{v}\right)=v_{U}
$$

By solving (6) in both directions, apart from the solution $c(t)$, we also will obtain the points $v_{H}$ and $v_{S}$ where $c(t)$ intersects $H$ and $S$ respectively and the corresponding values for the parameter $t$ :

$$
\begin{gathered}
c(0)=v_{S}, v_{S} \in S \\
c\left(t_{\text {max }}\right)=v_{H}, v_{H} \in H
\end{gathered}
$$

Thus we propose to represent any point $v_{U} \in U$ by the point $v_{S} \in S$ and the normalized distance $d\left(v_{U}\right)$ along the corresponding integral curve $c(t)$ :

$$
d\left(v_{U}\right)=\int_{0}^{t_{v}}\left\|\frac{d c(t)}{d t}\right\| d t / \int_{0}^{t_{\max }}\left\|\frac{d c(t)}{d t}\right\| d t
$$

Having calculated the parameterization of $S$ and the normalized distance $d\left(v_{U}\right)$, and assuming $\varphi\left(v_{S}\right)=(\xi, \zeta)$, then every point $v_{U} \in U$ can represented inside the cylinder $v_{C}=\left(\xi, \zeta, d\left(v_{U}\right)\right) \in C$. If necessary, the same point can also be represented using cylindrical coordinates. 


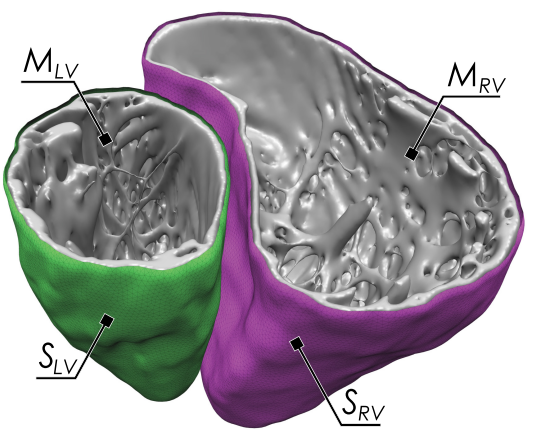

Figure 3: Visualization of detailed anatomical meshes and their extracted anatomical bounding surface meshes. The bounding surfaces are concave hulls of the trabeculated layer.

\section{Geometry independent representation of the cardiac ventricles}

The motivation for development of the proposed method was the parameterization of the cardiac ventricles for the representation of the detailed ventricular anatomy of the heart (Fig. 3).

The following subsections will explain how the proposed mapping framework can be applied to two meshes, $M_{L V}$ and $M_{R V}$ (in this paper - triangulated surface meshes), that represent detailed endocardial structures of the LVs and RVs, respectively. The outer bounding surfaces $S_{L V}$ and $S_{R V}$ are the corresponding anatomical bounding surface meshes extracted from $M_{L V}$ and $M_{R V}$. We define the anatomical bounding surface as the concave hull of the cardiac structures that we want to map onto the geometry independent representation. The process of obtaining meshes $M_{L V}, M_{R V}, S_{L V}$ and $S_{R V}$ is described in chapters 4.2.2 and 4.2.3.

Depending on the application, detailed anatomy meshes $M_{L V}$ and $M_{R V}$ can represent just endocardial structures of interest or all the structures within the ventricular cavity, and can include a part or the whole myocardial wall, therefore different anatomical boundary meshes $S_{L V}$ and $S_{R V}$ can be used, representing different meaningful anatomical bounding surfaces such as endocardium or epicardium.

For the interior surface $H$ we use a hemisphere of $5 \mathrm{~mm}$ radius located at the centroid of mitral(tricuspid) annulus. The choice of the centroid of the mitral (tricuspid) annulus as the origin and the size of the interior surface $H$ is motivated by the fact that this area is free of any detailed structures we 


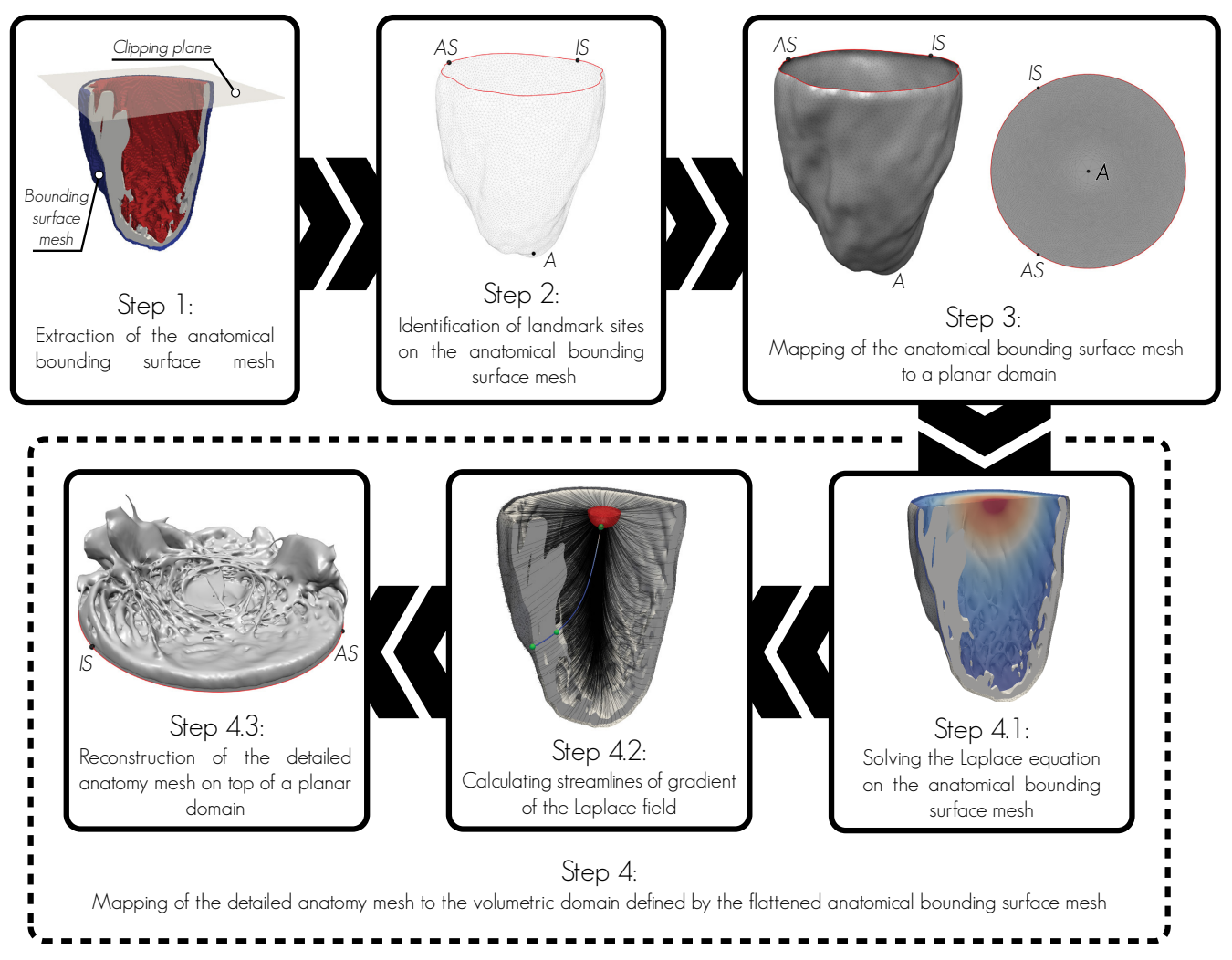

Figure 4: Pipeline of proposed method for generation of patient independent representation of ventricles. Landmarks: IS - inferoseptal, AS - anteroseptal, A - apex.

want to parameterize. The whole pipeline can be seen in the Fig. 4.

\subsection{Specification of the Landmark Points}

We propose to use the following landmarks for the ventricles: the ventricular apices and two points on the boundary of the septal wall that correspond to the locations where RV is attached to LV. Specifically, we manually define two landmark points $P_{L R V}$ and $P_{R R V}$ at the basal part of the RV ridges which divide the wall into lateral and septal part while all other landmarks are located automatically. From the two manually selected landmark points and the centroid of the LV boundary edge $\partial S_{L V}$ two vectors are constructed and their intersections with $S_{L V}$ are calculated. As those vectors do not necessarily intersect $S_{L V}$ at $\partial S_{L V}$ we locate closest points to intersections 


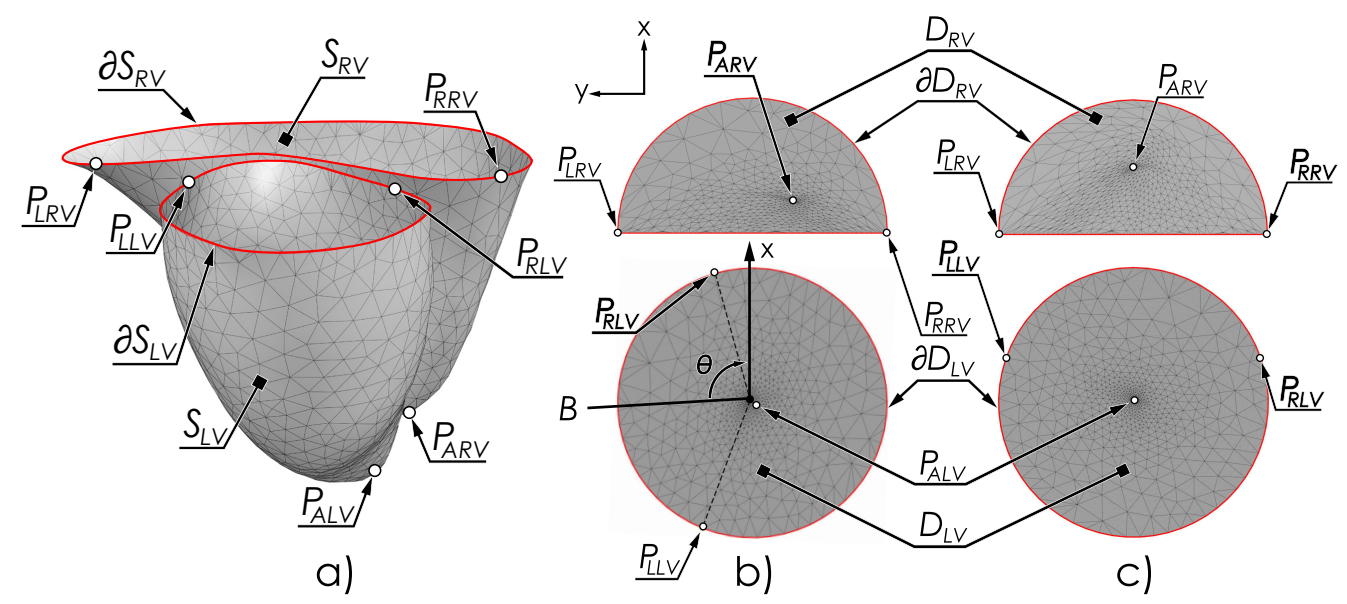

Figure 5: Illustration of the mapping procedure of the anatomical bounding surface meshes $S_{L V}, S_{R V}$ to the planar domains $D_{L V}, D_{R V}$ and alignment of the landmarks in the planar domain. a) Meshes $S_{L V}, S_{R V}$ with their corresponding landmarks. b) Meshes $S_{L V}, S_{R V}$ mapped to a planar domains $D_{L V}, D_{R V}$. c) Alignment of the landmarks to predefined places on planar domain.

points on $\partial S_{L V}$ and use them as the landmarks $P_{L L V}$ and $P_{R L V}$. Both apical landmarks $P_{A R V}$ and $P_{A L V}$ are calculated as the furthest points from the corresponding centroids of the anatomical boundary mesh edges $\partial S_{R V}$ and $\partial S_{L V}$. All the landmarks can be seen in Fig. 5 .

\subsection{Mapping of the Anatomical Bounding Surface Meshes to the Planar Do- main}

We chose the following planar domains for the bounding surfaces of the ventricles based on their typical representation encountered in the medical literature: $S_{L V}$ is mapped to a unit disk $D_{L V}$ and $S_{R V}$ to half of another unit disk $D_{R V}$ where their corresponding boundary edges $\partial S_{L V}$ and $\partial S_{R V}$ are mapped to $\partial D_{L V}$ and $\partial D_{R V}$ (Fig. 5b).

However, it is in our interest to map the different subjects' cardiac landmarks $P_{L L V}, P_{R L V}$ and $P_{A L V}$ to the same predefined locations on the disk. Thus after mapping $S_{L V}$ to a unit disk $D_{L V}$ (centered at the origin), we calculate the bisector of the angle between the center of the disk and the two septal landmarks $P_{L L V}$ and $P_{R L V}$. Subsequently all the points are rotated to align the bisector with the positive sense of the axis $\mathrm{x}$. The result is shown in 
Fig. 5b. After correcting the orientation we fit the Thin Plate Splines (TPS) to the interior vertices of $D_{L V}$ to displace the apical point $P_{A L V}$ to the center of the disk, leaving the boundary $\partial S_{L V}$ vertices intact. The results of the above mentioned alignment of the landmark points can be seen in Fig. 5c.

The half disk shape $D_{R V}$ was constructed from the unit circle centered at $(1.25,0)$ (this point was chosen for a convenient visualization of both ventricles, assuming $D_{L V}$ is at $\left.(0,0)\right)$, using only the points whose $x$ coordinates satisfy $x \geq 1.25$. The procedure of mapping $S_{R V}$ is the same as for $S_{L V}$ except the steps of alignment of landmarks and defining Dirichlet conditions. In this case we place two septal landmarks $P_{L R V}$ and $P_{R R V}$ at the corners of the half disk, namely $P_{L R V}=(1.25,1)$ and $P_{R R V}=(1.25,-1)$. The apex is again displaced using TPS to a predefined location $P_{A R V}=(1.75,0)$ (the point in the middle of the lateral and septal boundaries).

\subsection{Improved visualization of LV structures}

From the Fig. 5b and Fig. 5c we can see that the generated mapping increases the density of the vertices of $D_{L V}$ towards the apex which is visually unpleasant and hampers visual analysis of the information close to the apex. Thus, purely for visualization and for better correspondence of our representation to the AHA regions (Cerqueira et al., 2002), we radially displaced the interior vertices of $D_{L V}$ using the following equations:

$$
\begin{aligned}
x^{\prime} & =\left(x^{2}+y^{2}\right)^{n / 2} \cos \left[\tan ^{-1}(y / x)\right] \\
y^{\prime} & =\left(x^{2}+y^{2}\right)^{n / 2} \sin \left[\tan ^{-1}(y / x)\right]
\end{aligned}
$$

where $(x, y)$ and $\left(x^{\prime}, y^{\prime}\right)$ are the original and modified vertex coordinates. For the value of exponent $n$ we chose $1 / 3$, which results in a visually more homogeneous distribution of information. Fig. 6 illustrates how points at different geodesic distances from the apex are mapped to the flat domain in the case of $n=1$ and $n=1 / 3$. The colors represent different regions of geodesic distance field from an apex calculated on $S_{L V}$, Fig. 6a, Fig. $6 \mathrm{~b}$ shows how the distances along the geodesics reduce towards the center, which is corrected in Fig. 6c using (11).

\subsection{Mapping of the Detailed Anatomy to Cylinder}

After obtaining $\varphi$, that maps meshes $S_{L V}$ and $S_{R V}$ to the corresponding planar domains, we proceed with mapping of the detailed anatomy meshes $M_{L V}$ and $M_{R V}$ to the cylinder defined by the corresponding planar domain as its base. 


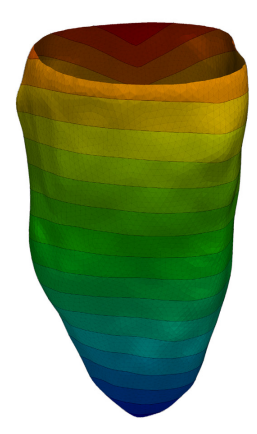

a)

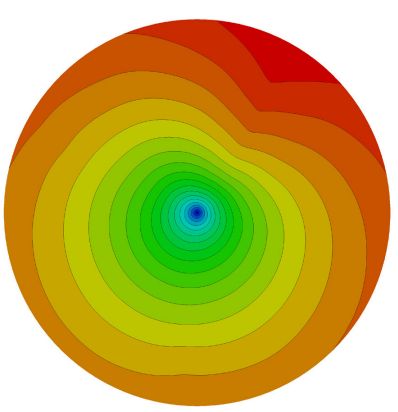

b)

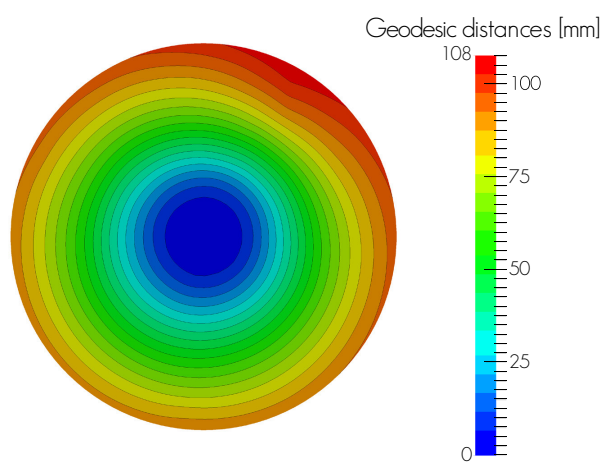

c)

Figure 6: Radial displacement of the vertices of $D_{L V}$ for better visualization and correspondence with AHA segments. Colors represent different regions of geodesic distance field from an apex calculated on $S_{L V}$. a) Mesh $S_{L V}$ with calculated geodesic distance field and its isolines. b) Mesh $D_{L V}$ before radial displaced of its inner vertices. c) Mesh $D_{L V}$ after radial displacement of its inner vertices.

The whole procedure is summarized in Algorithm 1, where the enclosing surface $S$ and inner surface $H$ are represented by triangulated meshes. The resulting points $v_{N}$ define the parameterized surface $N$ - a shell-like object mapped to the reference frame. The application of the Algorithm 1 to the $M_{L V}$ is illustrated in Fig. 7.

\subsection{Inverse Mapping and Mapping to a Different Geometry}

We require our mapping to be bijective, thus we have to make sure that its inverse exists. To map the parameterized mesh $N$ back to its original shape or any other, we employ the procedure described in Algorithm 2, which simply reverses the steps of the Algorithm 1.

\section{Datasets}

\subsection{Synthetic data}

For synthetic data we generated tubular structures with two different regular patterns, circumferential and longitudinal (Fig. 8), similar to Karim et al. (2014).

Circumferential tubes (Fig. 8a) are created by clipping the scaled down version of the anatomical bounding surface mesh $S$ by equally spaced planes, 

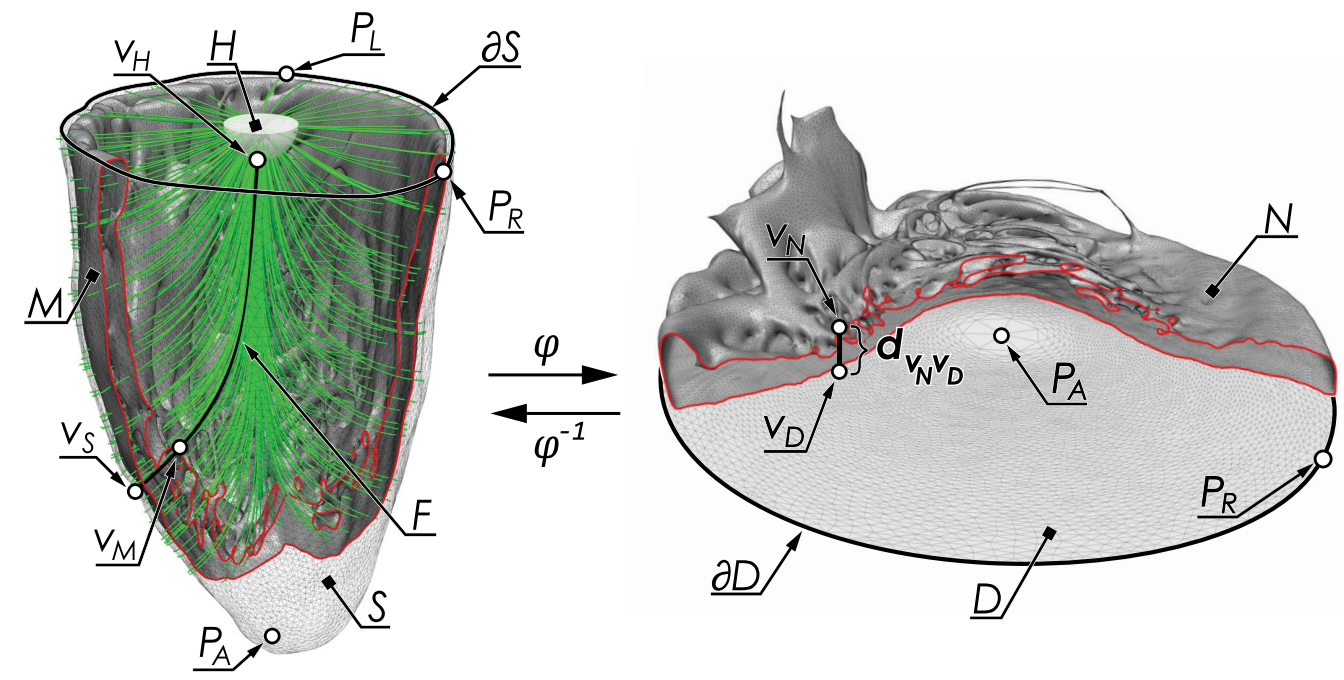

Figure 7: Illustration of a mapping procedure of a detailed anatomical LV mesh vertex $v_{M}$ to the patient independent reference frame.

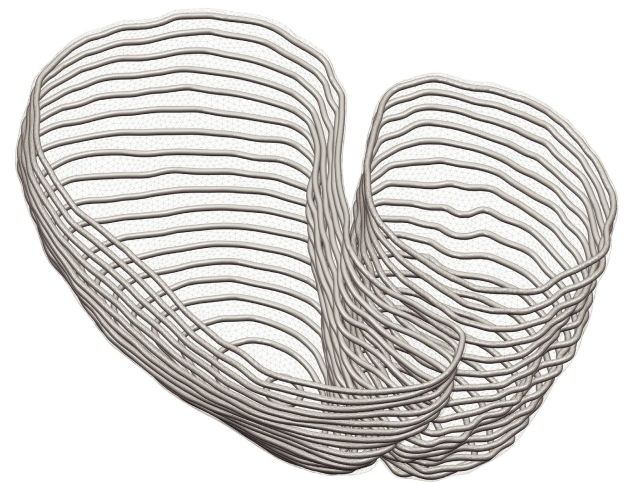

a)

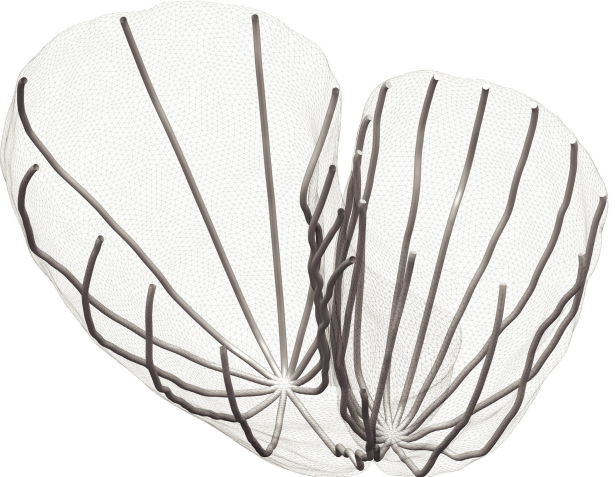

b)

Figure 8: Illustration of two different patterns of tubular structures representing synthetic data. a) Circumferential tubes. b) Longitudinal tubes.

that are orthogonal to the long axis. The curves obtained by the clipping were then uniformly subdivided and used as a centerlines of tubular structures. The radius of the tubes was set to $0.7 \mathrm{~mm}$. 


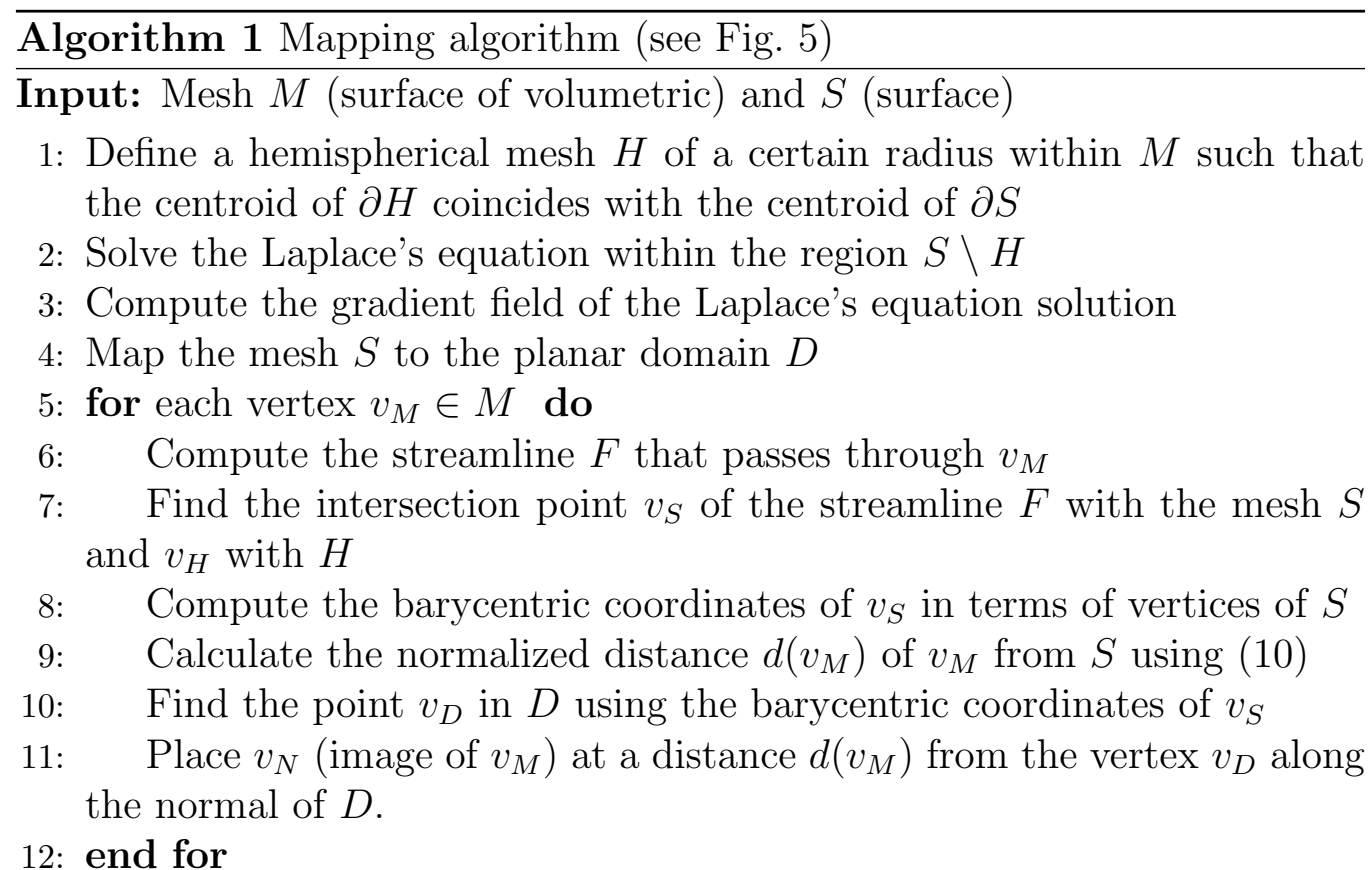

Output: Mesh $N$ consisting of vertices $v_{N}$ with the same connectivity as the mesh $M$.

Longitudinal tubes (Fig. 8b) are created by clipping the same meshes by a plane defined by the ventricle's apices and centroids of the boundary edges. The plane was rotated by 30 degree increments and the curves obtained by the clipping were used as the centroids of the tubes generated using the same parameters as the radial tubes.

\subsection{Human Hearts}

\subsubsection{Image Datasets}

We processed six MRI datasets acquired by a $3 T$ Siemens scanner with $0.44 \times 0.44 \mathrm{~mm}$ in-plane resolution and slice thickness of 1 to $1.17 \mathrm{~mm}$. The Dicom datasets (Fig. 9) were provided by the Visible Heart ${ }^{\circledR}$ Laboratory, and were obtained by MRI scanning perfusion fixed hearts that were graciously donated by the organ donors and their families through LifeSource. The aorta, trachea, superior vena cava, and the inferior vena cava (when possible) of a given specimen were cannulated and attached to a perfusion fixation chamber as described previously (Anderson et al., 2009, 2008; Eggen et al., 2012; Goff et al., 2015). This approach preserved/fixed each heart in 


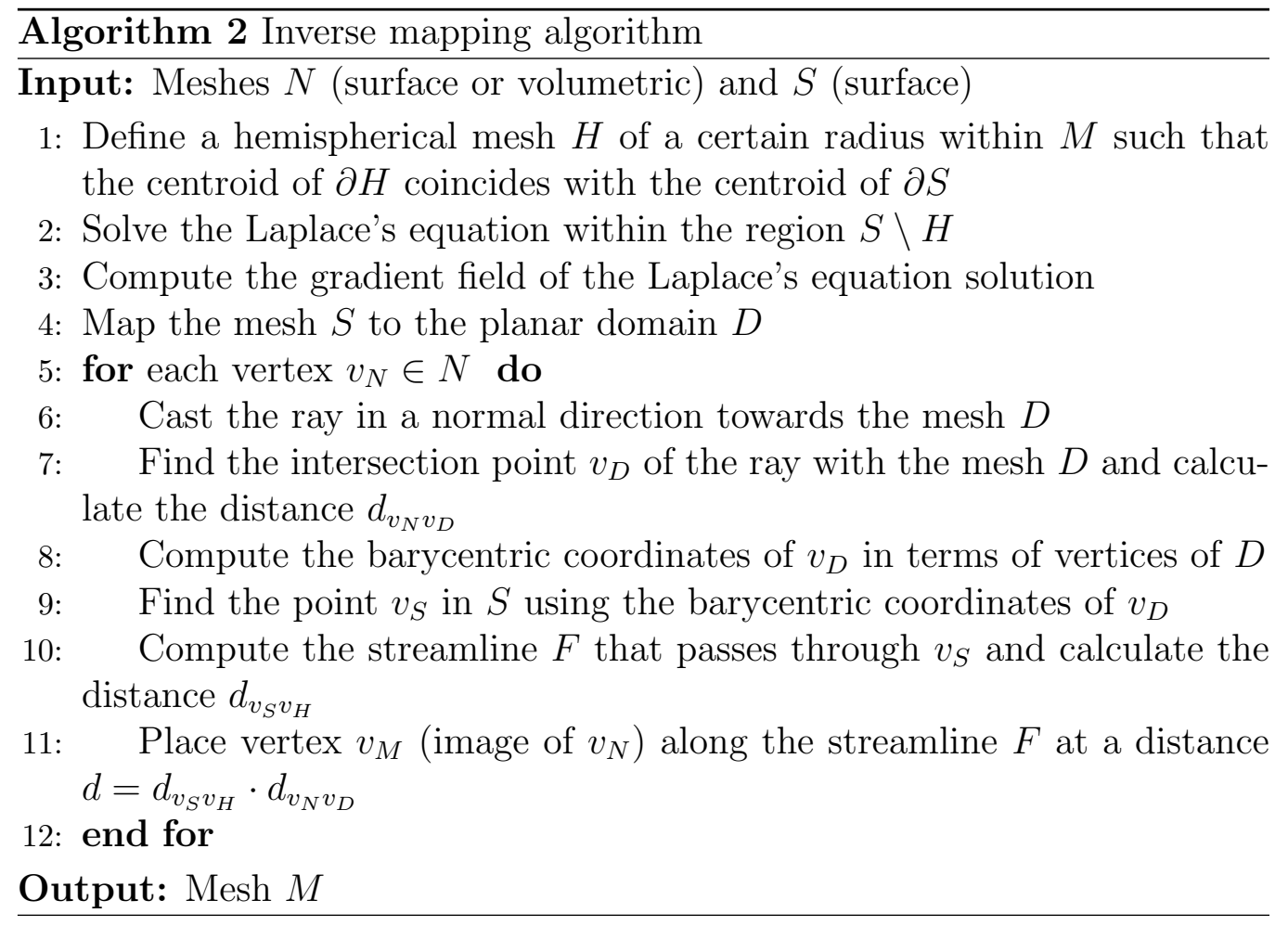

a modified end-diastolic state (atria and ventricles were fully expanded) and also lungs were dilated. The pericardium and phrenic nerves were left intact. These hearts were fixed with $10 \%$ formalin in PBS solution for at least 24 hours under $40-50 \mathrm{mmHg}$ of pressure, and then stored in $10 \%$ formalin.

\subsubsection{Segmentation}

The meshes of the detailed ventricular anatomy (Fig. 10) were generated from the segmentations of the MRI data with the Seg3D image processing software (CIBC, 2015). The segmentations were cut at the basal part of the heart with a plane perpendicular to the LV long axis immediately under the mitral valve and the supraventricular crest. Tendinous chords as part of the valves protruding into ventricular cavities of heart ventricles were removed during the segmentation process of the ventricles. As we are focusing only on the trabeculations, the myocardium was excluded from the segmentations using the following procedure. First the blood pool was segmented and morphological dilation-erosion (Seg3D, CIBC (2015), kernel size $12 \mathrm{~mm}$ ) is applied to obtain an approximation to the concave hull of the tra- 


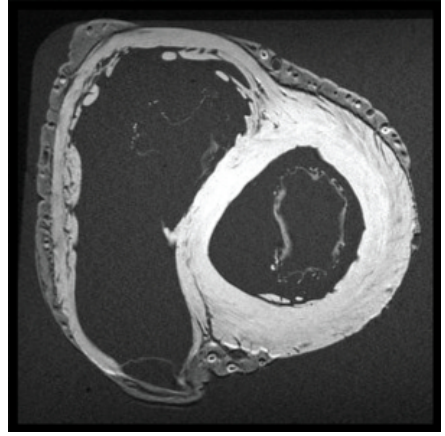

(a) Dataset HH 84

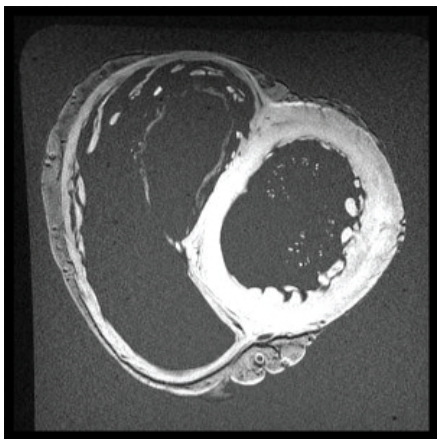

(c) Dataset HH 111

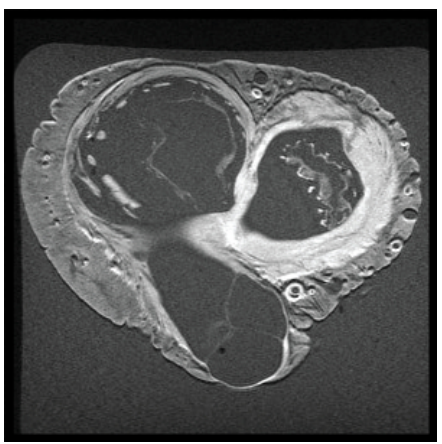

(e) Dataset HH 119

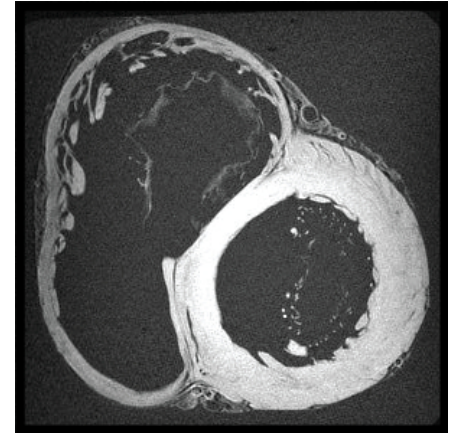

(b) Dataset HH 88

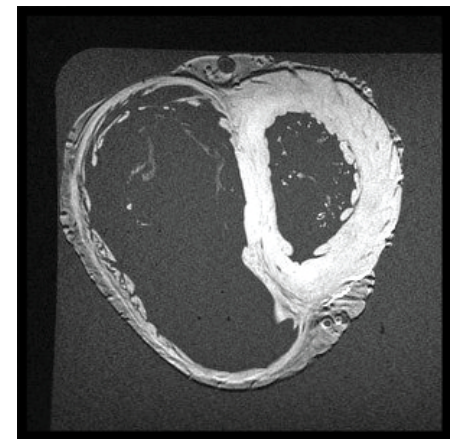

(d) Dataset HH 112

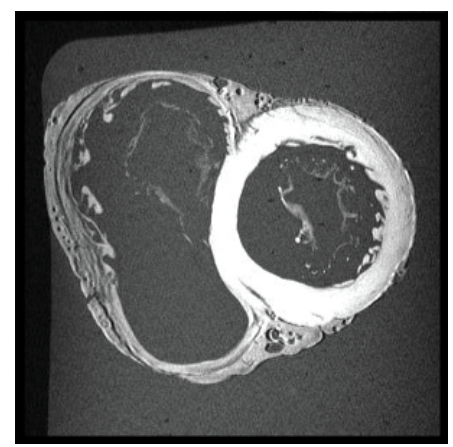

(f) Dataset HH 121

Figure 9: Middle stack short axis view slices of MRI human heart datasets.

beculated endocardium. The blood pool image was then subtracted from the concave hull image to obtain final segmentation of detailed cardiac ventric- 
ular anatomy. The meshes of the segmentations were obtained in ParaView (Squillacote, 2008) and were smoothed and uniformly remeshed in ReMesh (Attene and Falcidieno, 2006). We used 1 iteration of uniform remesh filter and 3 iterations of Laplacian smooth filter.

\subsubsection{Extraction of the Anatomical Bounding Surface Meshes}

To be able to parameterize the detailed cardiac anatomy meshes $M_{L V}$ and $M_{R V}$, we need to define the domain for solving the Laplace equation in which those meshes will be embedded. The domain is defined by the anatomical bounding surface and the hemisphere placed at the centroid of the boundary of anatomical bounding surface (i.e. the centroid of the mitral/tricuspid valve in our case). From the meshes $M_{L V}$ and $M_{R V}$, we first create a binary volumetric images (1 - detailed cardiac anatomy, 0 - background) of isotropic resolution $0.5 \mathrm{~mm}$ and slightly dilate the images by a constant value of 1.0 $m m$ (Visualization Toolkit (VTK) Schroeder et al. (2006), kernel radius of 2 voxels). The dilation is required to avoid the intersection of this bounding surface, used to define the mapping, and the structures to be mapped. From the dilated images we extract the boundary surfaces by the Marching Cubes algorithm (Lorensen and Cline, 1987). The extracted surfaces are then smoothed with VTK's window sinc filter and uniformly remeshed.

The next step is to introduce a cut separating the extracted surface into two parts. The cut is introduced at the highest point of the basal rim of the extracted surface by clipping it with a plane orthogonal to the LV long axis, such that the surface is divided into two parts: an inner part, which is in contact with the blood pool and will be discarded, and an outer part, which will represent our enclosing surface $S$. (Fig. 4 Step 1). The outer part surface is then isolated and smoothed. The enclosing surfaces are then uniformly remeshed and taken as $S_{L V}$ and $S_{R V}$. Lastly we compute the boundaries (curves) $\partial S_{L V}$ and $\partial S_{R V}$ of $S_{L V}$ and $S_{R V}$.

\section{Results}

\subsection{Algorithm Performance}

Our mapping method relies on the solution of the Laplace's equation of discretized volumetric domain between the meshes $H$ and $S$. The streamlines of the gradient of the Laplace's equation solution allows us to map every point inside the discretized domain. Thus the error introduced by our mapping procedure will depend on the resolution of the discretized domain. The 


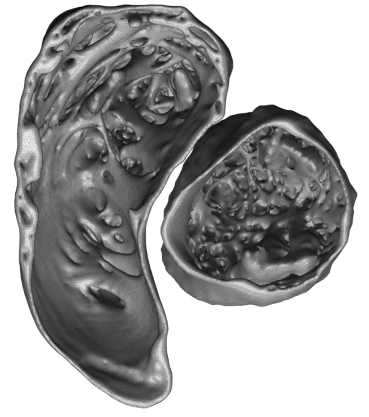

(a) HH 84 mesh

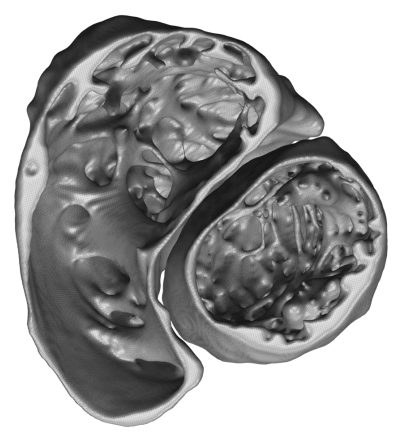

(c) HH 111 mesh

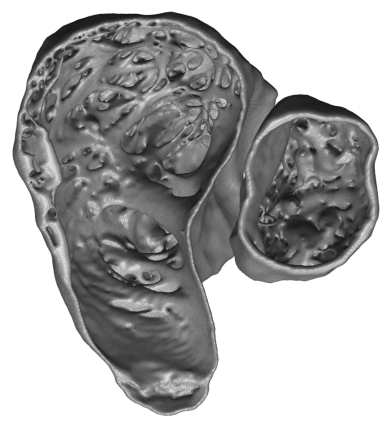

(e) HH 119 mesh

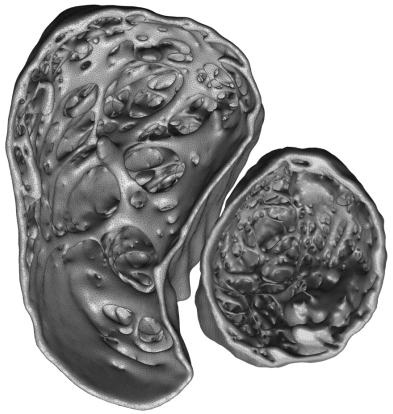

(b) HH 88 mesh

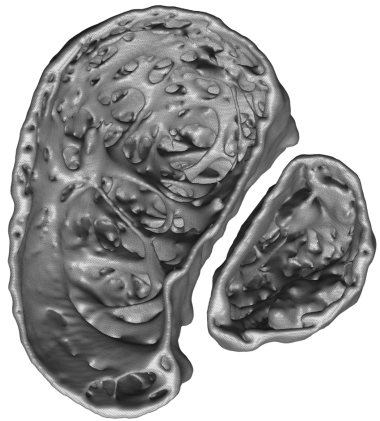

(d) HH 112 mesh

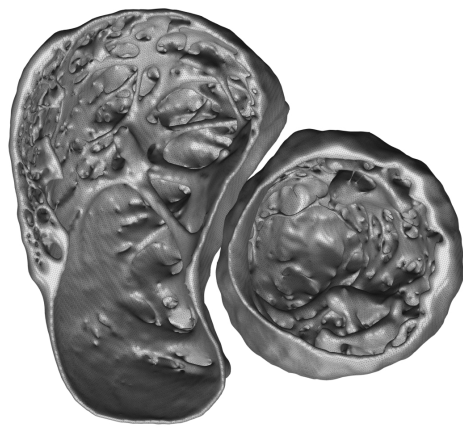

(f) HH 121 mesh

Figure 10: Meshes corresponding to the segmentations, representing only the trabeculated layer.

Laplace's equation was solved iteratively until the update between consecu- 


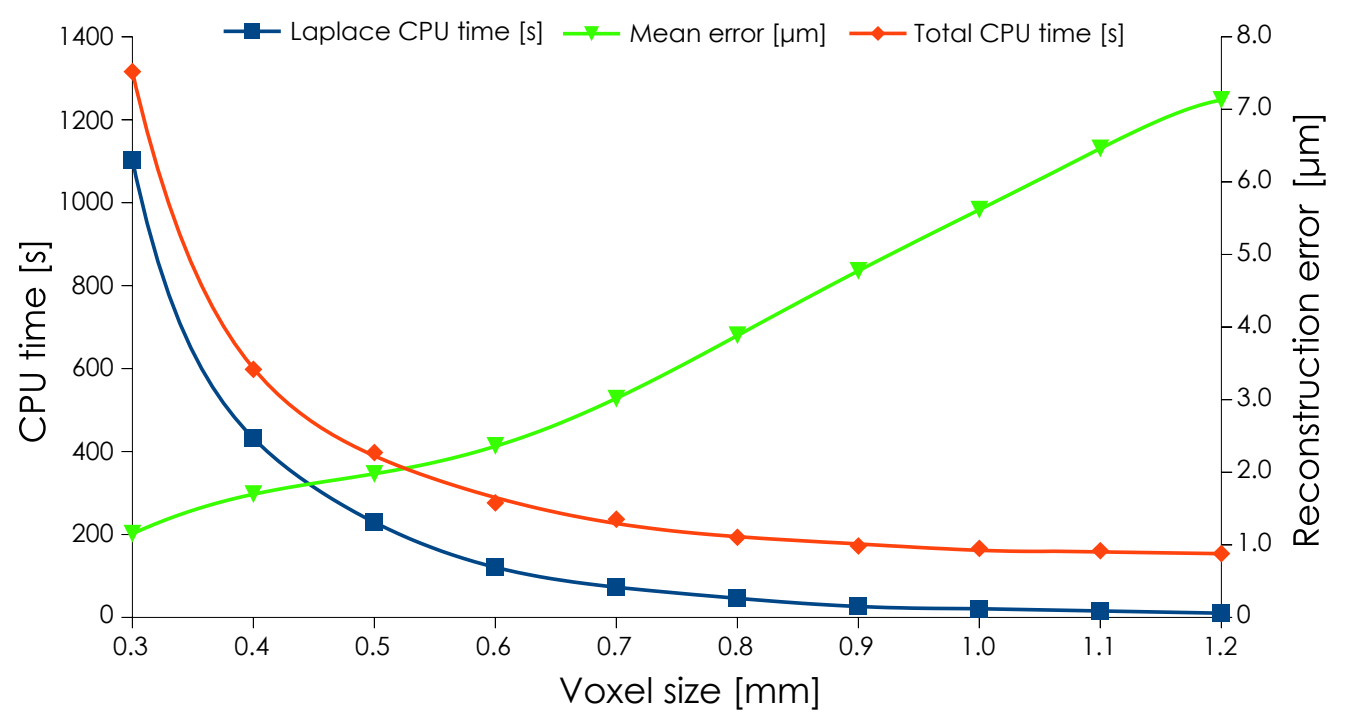

Figure 11: Reconstruction errors and execution times of the algorithm with respect to the size of the voxels.

tive iterations for every point of the domain was smaller than or equal to a predefined threshold $\epsilon=0.0001$. The execution time of our $\mathrm{C}++$ implementation of the mapping algorithm and its reconstruction error were executed on a SNOW Linux Cluster equipped with AMD Opteron Abu Dhabi 6378 processors.

We analyzed how the performance changes with the voxel size of the discretized domain and the relationship between the speed and the reconstruction accuracy. The experiment was carried out on the dataset $H H 111$ (see Fig. 9). The voxel sizes ranged between $0.3-1.2 \mathrm{~mm}$ in the steps of $0.1 \mathrm{~mm}$ where the maximum value was chosen to be slightly bigger than the maximum slice thickness of our datasets. The reconstruction error is defined as Euclidean distance between vertices of mesh $M$ and their corresponding vertices of a mesh $M$ that underwent direct and inverse transformation $\psi^{-1}[\psi(M)]$. In Fig. 11 we show the calculated reconstruction errors and execution times for the whole direct mapping procedure and the time necessary to calculate the solution of the Laplace's equation.

As expected, the reconstruction error increases with the increase of the voxel size of discretized domain while the time to obtain the map and the 

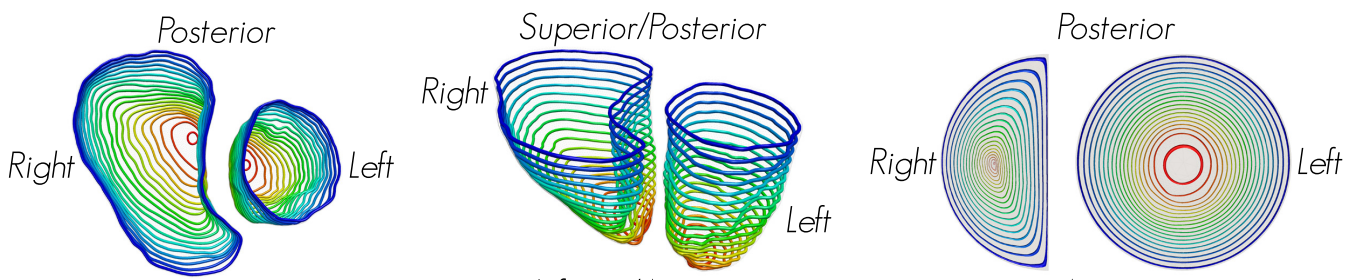

Anterior

Inferior/Anterior

Anterior
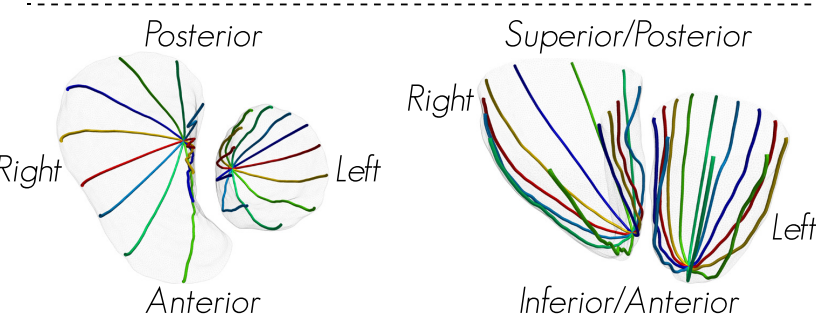

Posterior
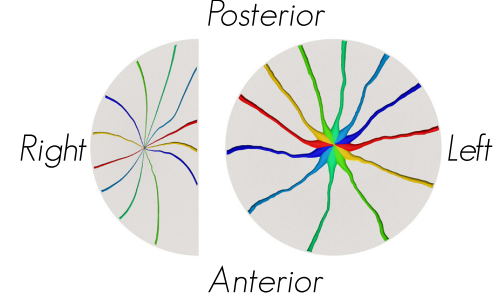

Anterior

Figure 12: Mapping of synthetic data. The synthetic tubular structures were colored differently to aid visualization. The circumferential tubes (top row) are colored according to their position along the long axis, while the longitudinal tubes (bottom row) are colored according to the angle of the defining plane.

time to solve the Laplace's equation decreases.

\subsection{Reconstruction Accuracy Measure}

In all the following experiments we measure the accuracy of our algorithm in terms of mesh reconstruction. The measurement is carried out by applying the direct and inverse transform $\psi$ to the mesh $M$ and calculating the average point to point distance between $M$ and $\psi^{-1}[\psi(M)]$.

\subsection{Analysis of the Distortion Caused by the Mapping}

\subsubsection{Distortion due to the Direct Mapping}

It is known that although the conformal flattening preserves angles locally, it will introduce some distortion globally. The distortion could also increase due to the use of the TPS for the left ventricle. In this experiment we wanted to assess visually the distortion introduced by the proposed method applying it to synthetic data - tubular structures with two different regular patterns: circumferential and longitudinal, similar to Karim et al. (2014). Both tubular structure arrangements were then mapped according to the proposed method. 
From the Fig. 12 one can observe that the more the radial or circumferential curvature of the bounding mesh $D$ deviates from the one of the ellipsoid the higher will be distortion in that direction, namely there will be radial or circumferential inhomogeneities in the planar domain. Circumferential inhomogeneity can be seen in the case of LV circumferential tubes. The mapped circumferential tubes generally nicely follow the perimeter of the disk, but when approaching the apex we can observe radial inhomogeneities due to higher deviation of the mesh curvature from the curvature of the ellipse. In the RV we can observe that the tubes nicely follow the shape perimeter in lateral part, while they get more stretched when approaching RV ridges (lines connecting the apex and the reference landmarks at shape corners). Also, there is higher distortion of the tubes in the septal part caused by the stretch of ventricular septal wall.

The longitudinally oriented tubes in LV and RV also slightly deviate from straight lines. Again, this is caused by regional deviations from an ellipsoid. Those inhomogeneities are more pronounced in the LV than in the RV because of the applied radial displacement of the vertices of the disk domain due to (11), thus artificially compressing the vertices towards the basal segments. The longitudinal tubes in the RV planar domain deviate from straight lines due to higher stretch of the surface towards the boundary. Such inhomogeneities are particularly visible in the septal part where certain tubes make sharper turns due to the high stretch of the basal part of the septal wall close to the boundary. As the vertices of RV are not displaced radially outwards (as was done in LV) the longitudinal tubes appear to be more smooth than in LV.

We would like to emphasize that, although the direct map can introduce above mentioned inhomogeneities, the introduced distortions will disappear when we map those structures back to the patient anatomy.

\subsubsection{Distortion due to Direct and Inverse Mapping}

Since the process of mapping involves discretization of the volumetric domain enclosed by the anatomical mesh $S$, we expect some error to be introduced by the discretization. In this experiment we analyze this distortion by calculating the direct and inverse transform and analyzing the reconstruction error for all the anatomical meshes (Fig. 10). Fig. 13 depicts the reconstruction errors.

To obtain the direct and inverse transform of the $M$ meshes we calculated the solution to the Laplace's equation using a threshold value $\epsilon=0.0001$ 


\begin{tabular}{|r|c|c|c|c|c|c|c|c|c|c|c|c|c|}
\cline { 2 - 13 } \multicolumn{1}{c|}{} & \multicolumn{2}{c|}{ HH84 } & \multicolumn{2}{c|}{ HH88 } & \multicolumn{2}{c|}{ HH111 } & \multicolumn{2}{c|}{ HH112 } & \multicolumn{2}{c|}{ HH119 } & \multicolumn{2}{c|}{ HH121 } & \multirow{2}{*}{ AVERAGE } \\
\cline { 2 - 13 } \multicolumn{1}{c|}{} & LV & RV & LV & RV & LV & RV & LV & RV & LV & RV & LV & RV & \\
\hline Mean distance $[\mu \mathrm{m}]$ & 1.24 & 1.75 & 1.28 & 1.31 & 1.15 & 1.70 & 1.84 & 1.40 & 1.84 & 1.50 & 1.21 & 1.56 & 1.48 \\
\hline Median distance $[\mu \mathrm{m}]$ & 0.80 & 1.15 & 0.81 & 0.90 & 0.85 & 1.16 & 1.23 & 0.92 & 1.25 & 0.97 & 0.70 & 1.02 & 0.98 \\
\hline Standard deviation $[\mu \mathrm{m}]$ & 1.78 & 2.13 & 1.86 & 1.64 & 1.81 & 2.11 & 2.21 & 1.76 & 2.16 & 1.86 & 1.95 & 2.05 & 1.94 \\
\hline
\end{tabular}

Table 2: Mean, median and standard deviation of the reconstruction errors for each mesh.

on the corresponding volumetric domains which we discretized to voxels of isotropic size of $0.3 \mathrm{~mm}$. This voxel size was chosen to have the highest resolution of the volumetric domain without increasing too much the computational requirements (going below $0.3 \mathrm{~mm}$ required significantly more computational resources and time).

The mean and median reconstruction error for each ventricle, together with their standard deviations, are shown in Table 2. The maximum reconstruction error between any given pair of vertices is $35.0 \mu \mathrm{m}$, while the average mean and median reconstruction errors are 1.40 and $0.88 \mu \mathrm{m}$ with the average standard deviation of $1.90 \mu \mathrm{m}$.

\subsection{Landmark Placement Sensitivity}

Our method relies on the selection of three landmark points on the anatomical bounding surfaces $S$, thus we investigated the impact of the inaccuracies of their localization. In the following experiments we used the reconstruction error, however the inverse map was calculated using the landmarks displaced with respect to the ones used for the direct transform. This approach simulates the scenario when two hearts are mapped to our patient independent representation with inexact landmark placement, except that since the same mesh is used, the change can be easily calculated.

We simulated three types landmark placement inaccuracies (in all the schemes the landmarks were displaced by fixed distances of 1,3 and $5 \mathrm{~mm}$ ):

- one of the boundary landmarks is displaced from the optimal position

- both boundary landmarks are displaced towards each other or in the opposite direction

- the apical landmark is displaced

In the first scheme, we displaced one boundary landmark at a time: $P_{L L V}$, $P_{R L V}$ on $S_{L V}$, and $P_{L R V}, P_{R R V}$ on $S_{R V}$ (LV and RV independently), while 

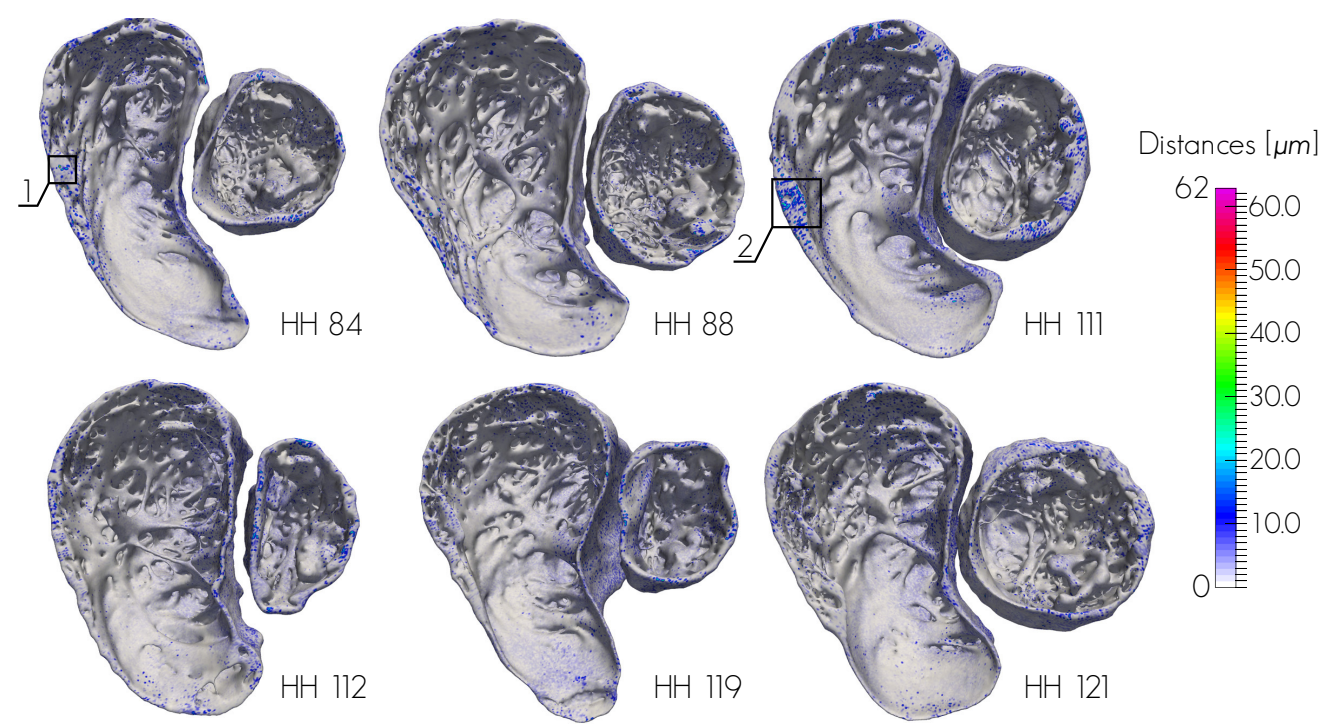

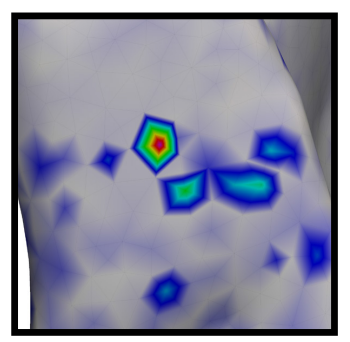

WINDOW 1

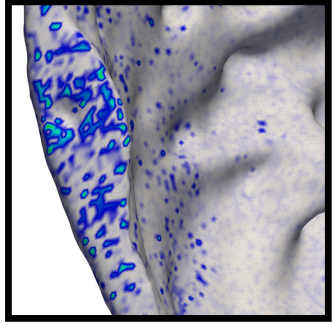

WINDOW 2

Figure 13: Reconstruction error per vertex. The reconstruction error is calculated as the vertex to vertex distance between original $M$ meshes and their corresponding instances after direct and inverse transform. The maximum was observed only in the RV mesh of $H H \quad 84$ dataset. Window 1 depicts the region of interest of the $H H 84$ dataset's RV, showing the location of the highest error. Window 2 is the closeup of the region of interest of $H H 111$ dataset's RV. One can notice that per vertex reconstruction errors of higher values are very localized over the meshes.

keeping the other two landmarks on $S$ fixed. Every moving landmark was displaced 1, 3 and $5 \mathrm{~mm}$ clockwise and counter-clockwise along the boundary $\partial S$.

The second scheme consisted of simultaneously moving both boundary landmarks: $P_{L L V}$ and $P_{R L V}$ on $S_{L V}$, and $P_{L R V}$ and $P_{R R V}$ on $S_{R V}$, while keeping the apical landmark fixed. The boundary landmarks were displaced 


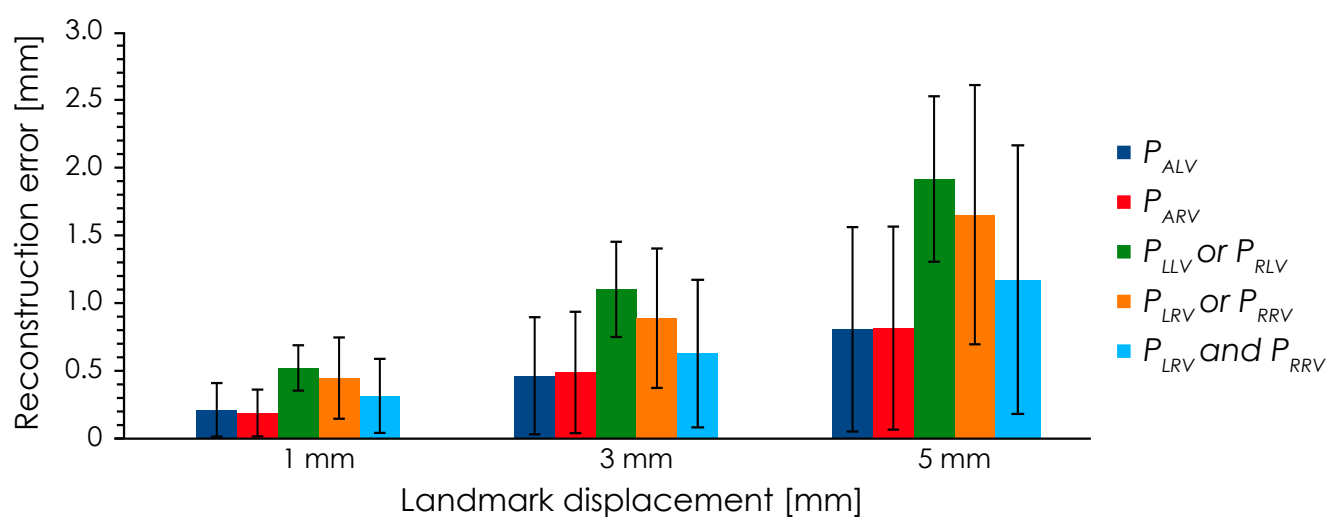

Figure 14: Average reconstruction errors with respect to the landmark displacement. Error bars represent one standard deviation. Plots labeled $P_{A L V}$ and $P_{A R V}$ show the reconstruction error when one of the apices is displaced; " $P_{L L V}$ or $P_{R L V}$ " and " $P_{L R V}$ or $P_{R R V}$ " correspond to the displacement of one of the boundary landmarks of RV and LV respectively; " $P_{L R V}$ and $P_{R R V}$ " corresponds to the displacement of both boundary landmarks of the RV.

simultaneously 1, 3 and $5 \mathrm{~mm}$ towards and away from each other along the boundary $\partial S$.

In the last scheme we displaced only the apical landmarks $P_{A L V}$ and $P_{A R V}$, while keeping the boundary landmarks fixed. These landmarks were displaced 1, 3 and $5 \mathrm{~mm}$ in four directions (orthogonal) along the surface.

Fig. 14 shows the average reconstruction error for different landmark displacement schemes and distances. As expected, the reconstruction error in both ventricles increases with the increase of the landmark displacement up to half the displacement magnitude.

Fig. 15 depicts the per vertex reconstruction error for a scheme where just the $P_{L L V}$ and $P_{L R V}$ were displaced counter clockwise along their boundaries. In the RV one can notice that the error is increasing both vertically from the apex $P_{A R V}$ and laterally around the wall from the $P_{R R V}$ towards the displaced landmark $P_{L R V}$. In the $\mathrm{LV}$ there is just vertical increase of the error from the apex $P_{A L V}$ towards $P_{L L V}$, while laterally around the wall the errors have similar values. From the figure we can observe that the error in the placement of one of the RV boundary landmarks leads to the inaccuracies limited to the neighborhood of the landmark. The neighborhood also increases with the magnitude of the displacement, affecting larger part of the RV with $5 \mathrm{~mm}$ 

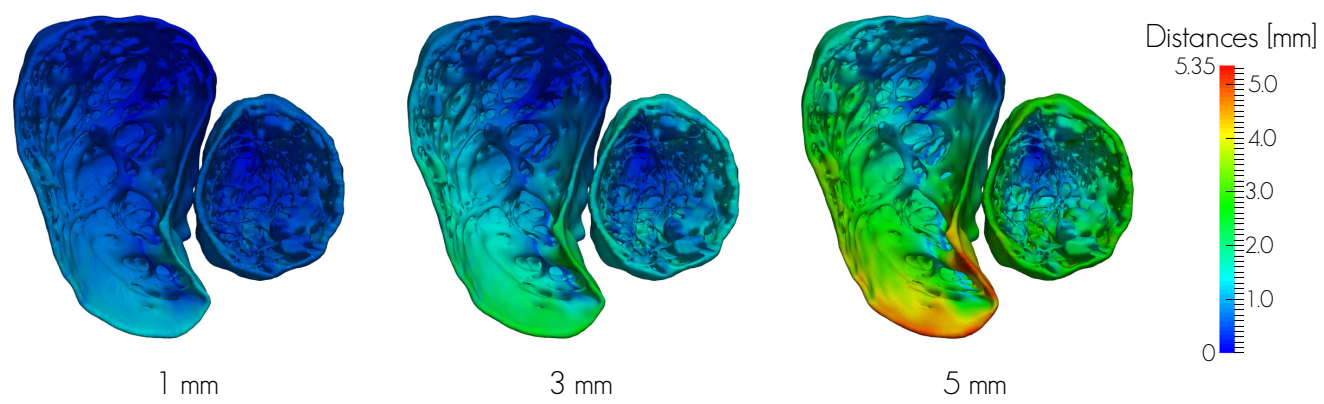

Figure 15: Reconstruction error per vertex for displacement of left boundary landmarks $P_{L L V}$ and $P_{L R V}$ for 1,3 , and $5 \mathrm{~mm}$ (view from the top, $H H 88$ dataset). Note the localized error in the RV and the uniformly spread errors in LV.

displacement. In the case of $\mathrm{LV}$, the change in one landmark $P_{L L V}$ changes the location of the bisector (see Section 3.2), which leads to the rotation of the ventricle. This rotation introduces radially uniform distortion, affecting mostly the structures in the basal parts of the LV.

In Fig. 16 we show per vertex reconstruction errors introduced by simultaneous displacement of the boundary landmarks $P_{L R V}$ and $P_{R R V}$ towards each other along the $\partial S_{R V}$. One can observe a vertical increase of the errors from the apex $P_{A R V}$ towards $P_{L R V}$ and $P_{R R V}$, while laterally they decrease around the wall from $P_{L R V}$ and $P_{R R V}$ towards the middle of septal and lateral wall. Again, we observe that the error in the placement of boundary landmarks leads to inaccuracies limited to the neighborhood of the landmarks, while the neighborhoods increase with the magnitude of the displacement.

In case of the LV, the visualization of the reconstruction errors on $H H$ 88 is omitted, as the left ventricles have shown invariance to this scheme of landmark displacement. The displacement of $P_{L L V}$ and $P_{L R V}$ gave the same result as for the case when landmarks are not displaced at all (Fig. 13). The invariance to the simultaneous displacement of $P_{L L V}$ and $P_{R L V}$ is caused by the fact that such displacement of the landmarks does not change the location of a bisector (see Section 3.2, Fig. 5), thus giving us the same reconstruction errors as in case of keeping the landmarks in place.

In the last displacement scheme, depicted in Fig. 17, we see that displacing the apical landmarks $P_{A L V}$ and $P_{A R V}$ cause the increase of per vertex reconstruction error towards the apex. As the boundaries are intact, there is 

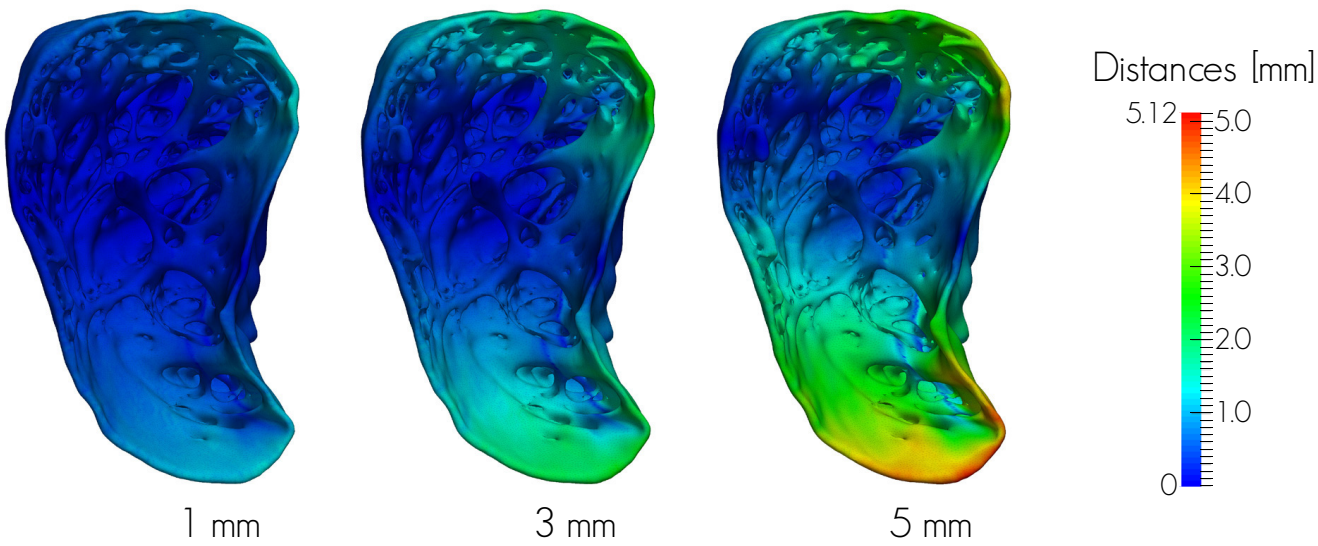

Figure 16: Reconstruction error per vertex for displacement of left and right boundary landmarks $P_{L R V}$ and $P_{R R V}$ simultaneously for 1, 3, and $5 \mathrm{~mm}$ towards each other (view from the top, $\mathrm{HH} 88$ dataset).

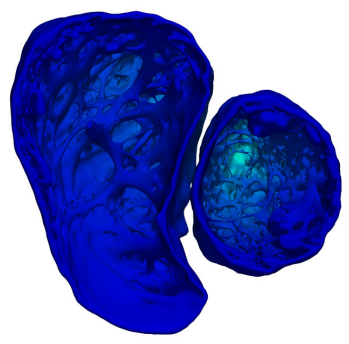

$1 \mathrm{~mm}$

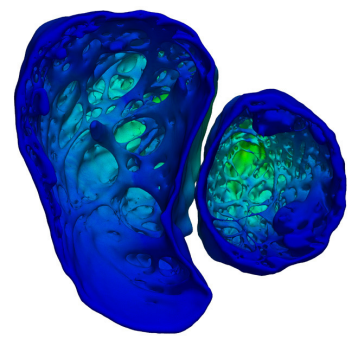

$3 \mathrm{~mm}$

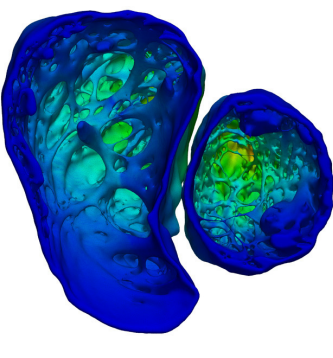

$5 \mathrm{~mm}$

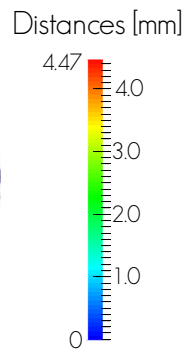

Figure 17: Reconstruction error per vertex for displacement of apical landmarks $P_{A L V}$ and $P_{A R V}$ for 1,3 , and $5 \mathrm{~mm}$ (view from the top, $H H 88$ dataset).

no change in the reconstruction errors in lateral direction. The placement of apical landmarks leads to the inaccuracies limited to the apical region, while the neighborhood is again increasing with the magnitude of the displacement.

\subsection{Application to the Human Hearts}

We applied our method to twelve detailed anatomy meshes $M$ (6 of right and 6 of left ventricle) and show their corresponding normalized anatomical meshes $N$ (subsection 5.5.1). As our mapping is bijective, it allows to map 
the $N$ meshes to any arbitrary shape represented by its anatomical bounding surface mesh $S$. Thus as an example in subsection 5.5.2 we show the results of mapping of the $N$ meshes to a hemisphere.

\subsubsection{Mapping of the Human Heart Datasets to the Normalized Anatomical Reference Frame}

The results of the proposed method are demonstrated on twelve extracted detailed anatomical meshes $M$ shown in Fig. 10. As mentioned in subsection 2.4, the RV detailed anatomical meshes were mapped to the normalized reference frame defined by half a cylinder of unit height and with the base being half the unit disk, while LV meshes are mapped to the volumetric domain defined by a unit cylinder, namely a cylinder of a unit disk base and unit height. The Fig. 18 depicts the obtained results.

In the Fig. 18 we can observe the intricate trabecular morphology inherent to each subject. An increase in the amount of trabeculations as we move from the base towards the apex can be seen in both ventricles. The basal part of the septal walls is free of trabeculations and they start to emerge in their middle parts while, on the lateral wall, we have them present along the whole wall. The trabeculations, present in the basal part of the lateral walls, are attached to the wall along their whole length and they form big prominent ridges. The moderator bands are clearly visible and traversing through the RV cavities. In the RV, there are more trabeculae traversing through the cavity and trabeculae emerging from opposite sides of the ventricle and fuse together to form the papillary muscles. The coarseness of trabeculations or "sponginess" of the heart increases towards the apex. That coincides with the observations reported in the literature of a highly trabeculated apical region where trabeculations form a complex interwoven network.

\subsubsection{Inverse Mapping and Mapping to Different Subject Geometries}

The proposed bijective map $\psi$ provides means to map any detailed anatomical mesh $M$ to a patient independent volumetric domain defined by the chosen planar domain as a base and unit height. Calculating such bijective map for different instances of the detailed patient anatomy allows us to map the anatomy of one patient to any other reference frame and vice versa.

The visualization in a volumetric domain with planar base may not be very visually pleasing due to trabeculas and moderator band traversing though ventricular cavities. Fig. 19 shows an alternative visualization, 


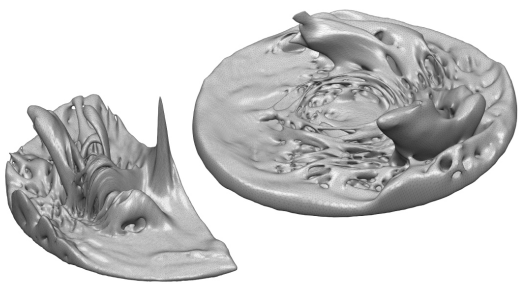

(a) HH 84 normalized

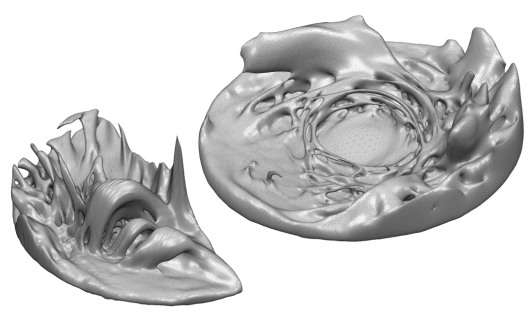

(c) HH 111 normalized

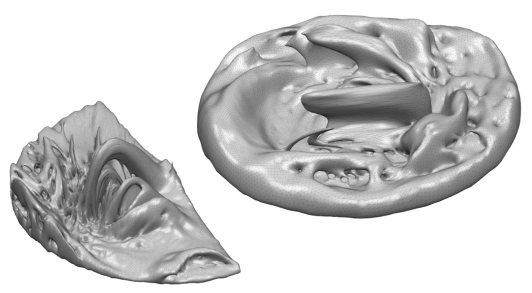

(e) HH 119 normalized

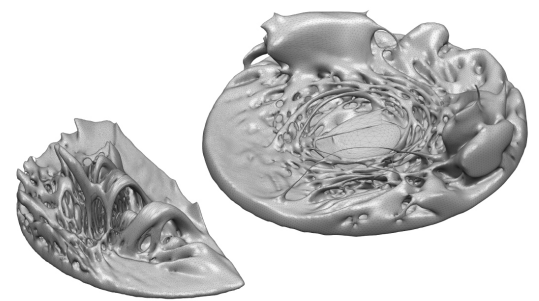

(b) HH 88 normalized

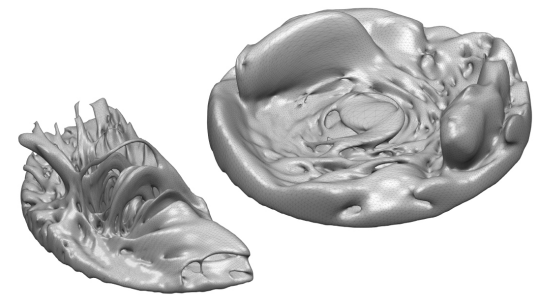

(d) HH 112 normalized

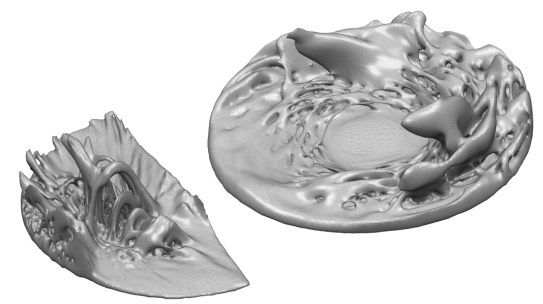

(f) HH 121 normalized

Figure 18: Detailed anatomical meshes $M$ mapped to the proposed normalized anatomical reference frame.

where the detailed anatomy meshes are mapped onto the domain defined by a hemisphere.

\subsubsection{Mapping of Human Heart Datasets Including Inflow and Outflow Tracts}

Throughout the paper we used the segmentations of the LV and RV detailed cardiac anatomy, which were cut by a plane perpendicular to the LV long axis immediately under the mitral valve and the supraventricular crest. However the proposed approach can also be used if the parameterization up to the inflow and outflow tracts is required (such as the one used in Young 


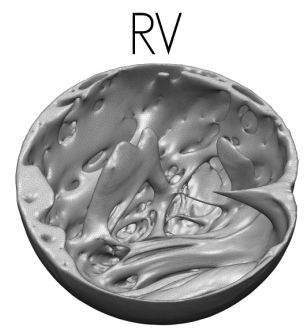

(a) HH 84 mesh
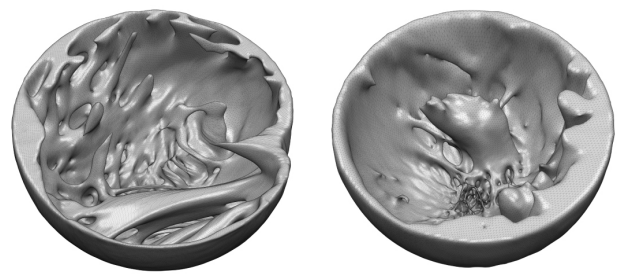

(c) HH 111 mesh
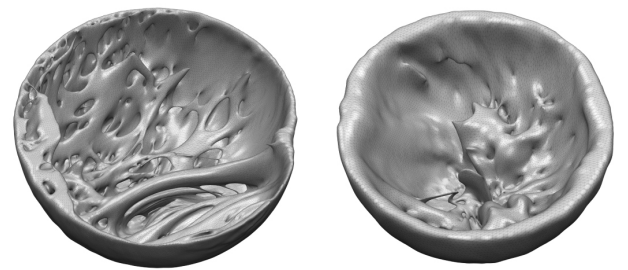

(e) HH 119 mesh
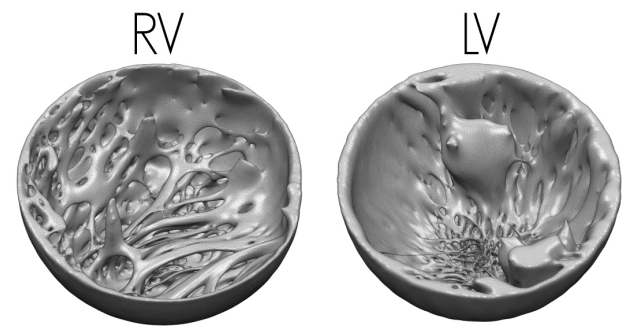

(b) HH 88 mesh
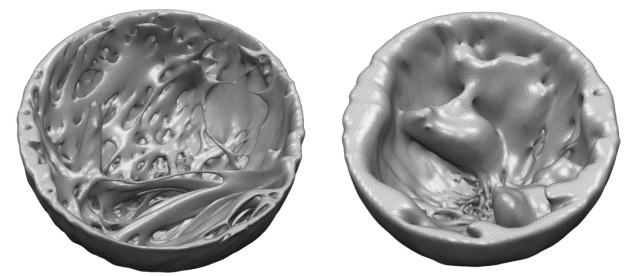

(d) HH 112 mesh
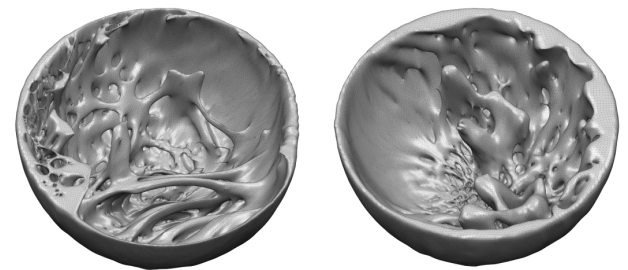

(f) HH 121 mesh

Figure 19: Mapping of detailed anatomical meshes $M$ to a hemisphere.

et al. (2011)).

In its proposed form, the parameterization is limited to geometries with only one opening. This limitation is based on the idea that such geometries can be conveniently flattened into a disk. However if more than one opening is present, as in the case of inflow and outflow tracts of the right ventricle (as in Gilbert et al. (2015)) the proposed approach can be modified in two ways: use one of the holes as the boundary and leave the other hole in the interior of the flat disk, similarly to Tobon-Gomez et al. (2015); or if the $2 \mathrm{D}$ visualization is not crucial, the parameterization can also be achieved by mapping the whole chamber to a spherical shell using exactly the same 


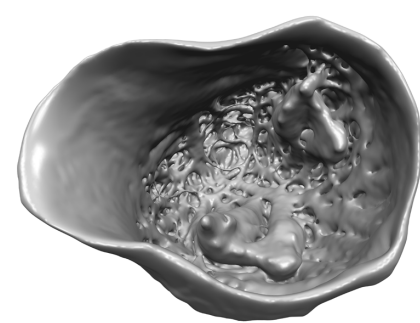

a)

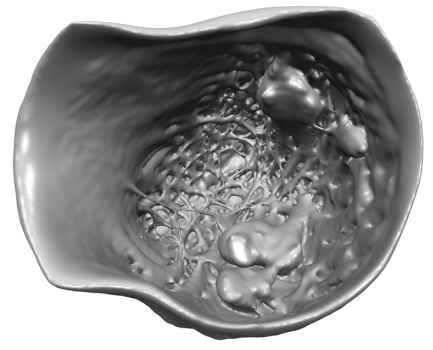

d)

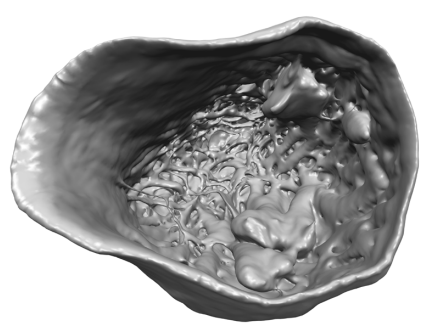

b)

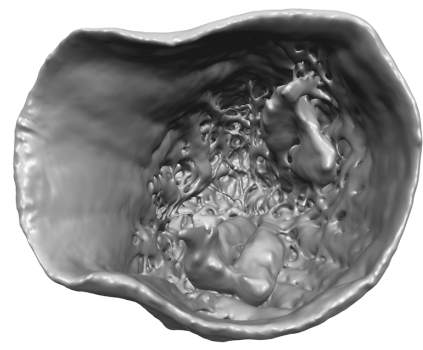

e)

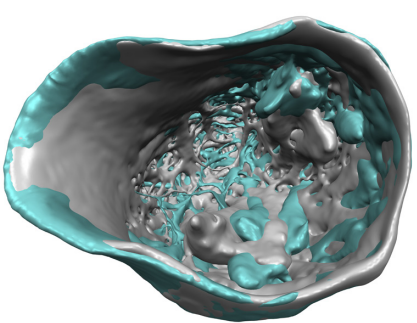

c)

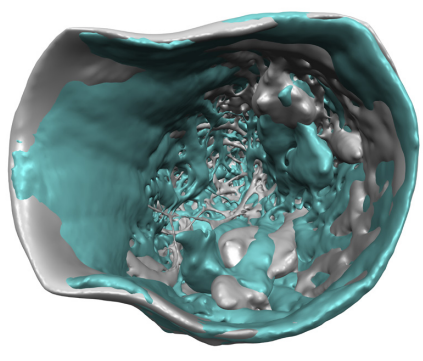

f)

Figure 20: Illustration of $\mathrm{LV}$ of $H H 84$ and $H H 88$ datasets including inflow and outflow tracts, and them mapped onto each other's bounding surface. a) Original $H H$ $84 \mathrm{LV}$ mesh. b)Mesh $H H 88 \mathrm{LV}$ mapped onto $H H 84 \mathrm{LV}$ bounding surface. c) Overlap of a)(gray color) and b)(turquoise color). d)Original $H H 88 \mathrm{LV}$ mesh. e)Mesh $H H 84 \mathrm{LV}$ mapped onto $H H$ $88 \mathrm{LV}$ bounding surface. f)Overlay of of d)(gray color) and e)(turquoise color).

methodology with an additional registration step to make sure the holes match on the sphere.

As an illustration, in Fig. 20 we show two LVs segmented up to the valves, and mapped to each other's bounding surface. Fig. 20a,d depict ventricles of $\mathrm{HH} 84$ and $\mathrm{HH} 88$ segmented up to the aortic and mitral valves. Their segmentations were cut with two planes placed thorough aortic and mitral annuli. Fig. 20b,e depict $H H 88 \mathrm{LV}$ mapped to $H H 84 \mathrm{LV}$ and vice versa, while Fig. 20c,f displays the overlap of Fig. 20a (gray color) with Fig. 20b (turquoise color) and Fig. 20d (gray color) with Fig. 20e (turquoise color).

\subsubsection{Relationship to the Bulls-eye Plot}

In this section we discuss the relationship of the proposed mapping to a bulls-eye plot using the 17 AHA segments (Fig. 21). For the illustration we partitioned the LV meshes of the datasets $H H 84$ and $H H 88$ (Fig. 22 left 


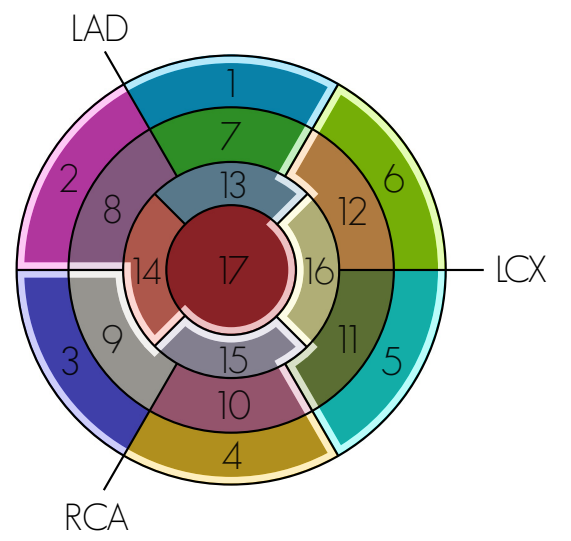

1. Basal anterior

7. Mid anterior

2. Basal anteroseptal

8. Mid anteroseptal

3. Basal inferoseptal

4. Basal inferior

9. Mid inferoseptal

5. Basal inferolateral

10. Mid inferior

6. Basal anterolateral

11. Mid inferolateral

13. Apical anterior

14. Apical septal

15. Apical inferior

16. Apical lateral

12. Mid anterolateral

17. Apex

Figure 21: Illustration of 17 AHA segments and the coronary artery regions with corresponding names and colors used in the text.

side) into 17 segments using the method proposed by Cerqueira et al. (2002) and our method:

- Cerqueira with equal partition. The first and fourth row of Fig. 22 represent the segmentation into 17 segments obtained by equal partitioning as proposed by Cerqueira et al. In this case, the ventricle is divided into 3 equal regions between mitral valve plane and the end of a cavity. The apical cap is defined as the area beyond the end of the cavity. The slices were defined by a plane perpendicular to the long axis (the line from the apex to the center of the mitral valve). The basal and mid-cavity slices were then divided into 6 equal segments of $60^{\circ}$. The first plane is defined by the long axis and the point in mid septum, while the other two are rotated $60^{\circ}$ with respect to it. The 4 segments of the apical slice are defined by 2 orthogonal planes, where the first one makes $45^{\circ}$ with respect to the mid septum.

- Cerqueira partitions using landmarks. The second and fifth row in Fig. 22 represent the ventricular segments defined using an alternative definition by Cerqueira et al., via specific landmark points. Basal, midcavity, and apical vertical long axis slices were then defined by a plane perpendicular to the long axis: the basal slice was defined as the area from the tips of the papillary muscles to the mitral annulus; the midcavity was defined as the region that includes the entire length of the 
papillary muscles; the apical slice was defined from bellow the roots of the papillary muscles to just before the cavity ends; and the apical cap was defined as the area of beyond the end of the left ventricular cavity.

The six myocardial segments of basal and mid-cavity slices are then defined by three planes. The first plane is defined by the long axis and a point where the right ventricular wall attaches to left ventricular wall in the anterior side of a heart. The second plane is defined in the same way except the point is located at the heart's inferior side. The third plane is defined by the long axis and a point located radially between the first and second plane.

The four apical slice segments are defined by two orthogonal planes passing though the long axis. The first plane is defined as a plane rotated $45^{\circ}$ from the mid septum and the second is orthogonal to the first.

- Equally sized regions in the flattened domain. The third and sixth rows of Fig. 22 depict the partition of the datasets into 17 segments obtained by our method. First we map the datasets to our proposed reference system and then transfer the segment labels information from the disk subdivided into regions as in Fig. 21. The labels are then mapped back to the original reference mesh and displayed.

The right side of Fig. 22 shows $H H 84$ and $H H 88$ LVs labeled using the above methods and mapped onto each other's bounding surfaces.

Strictly following the landmark information proposed by Cerqueira et. al. for defining the planes can be ambiguous, especially in case of high resolution images. As the roots and the tips of the papillary muscles are not located in the same plane perpendicular to the long axis, positioning of those planes is ambiguous and will lead to highly nonuniform longitudinal length of the regions. Such result can be seen in the second and fifth row of Fig. 22. Furthermore, defining the roots of the papillary muscles is subject to variability due to the fact that there are many trabeculations, which merge together to form the papillary muscles at different levels.

The planes separating the septum from the LV anterior and inferior free walls are defined by the long axis and the location of attachment of the RV wall to the LV. Those locations vary along the long axis and will lead to the nonuniform definition of radial basal and mid-cavity segments. 

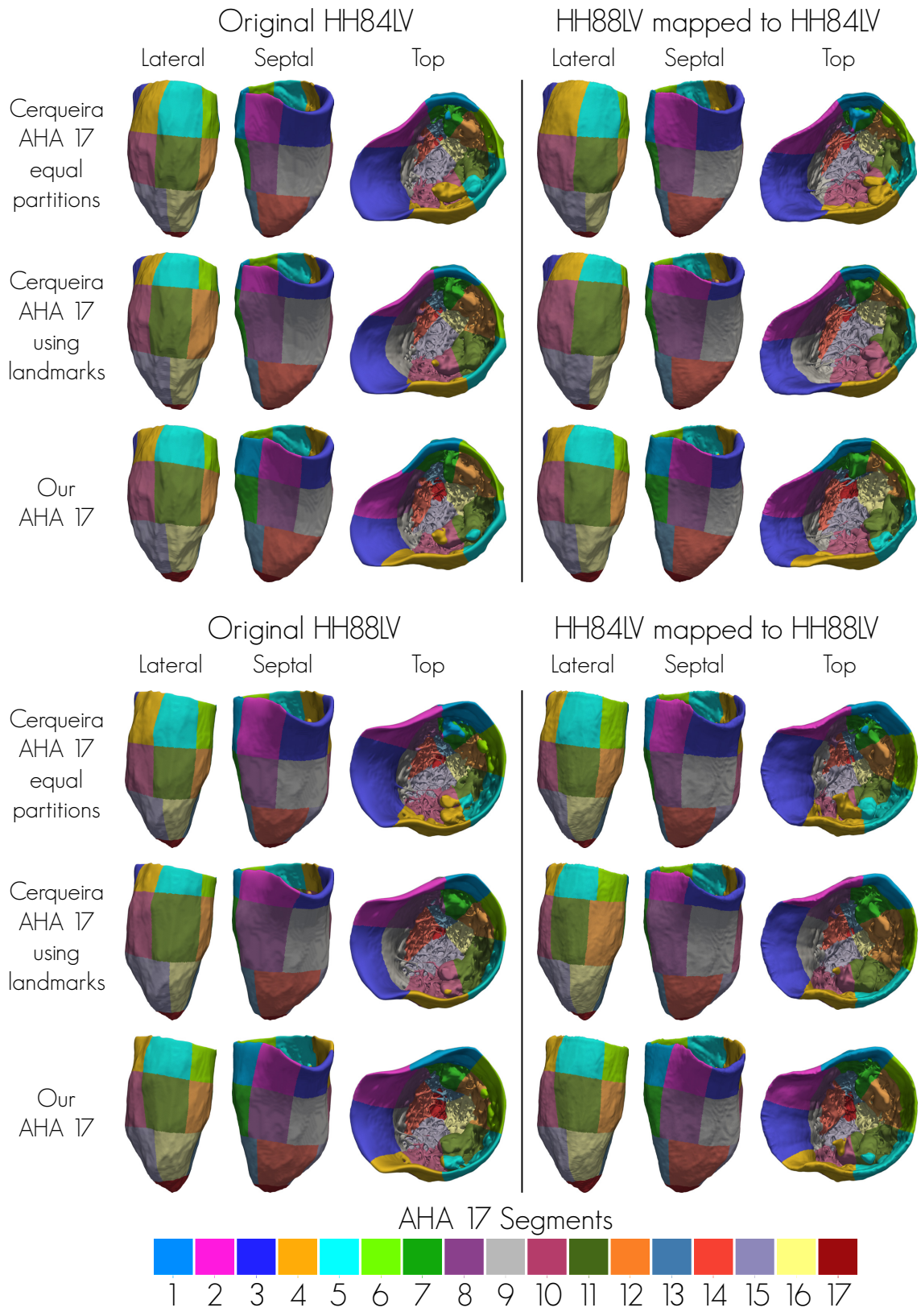

Figure 22: Partition of HH84 and HH88 LV meshes into 17 AHA segments and them mapped onto each other's bounding surfaces. 


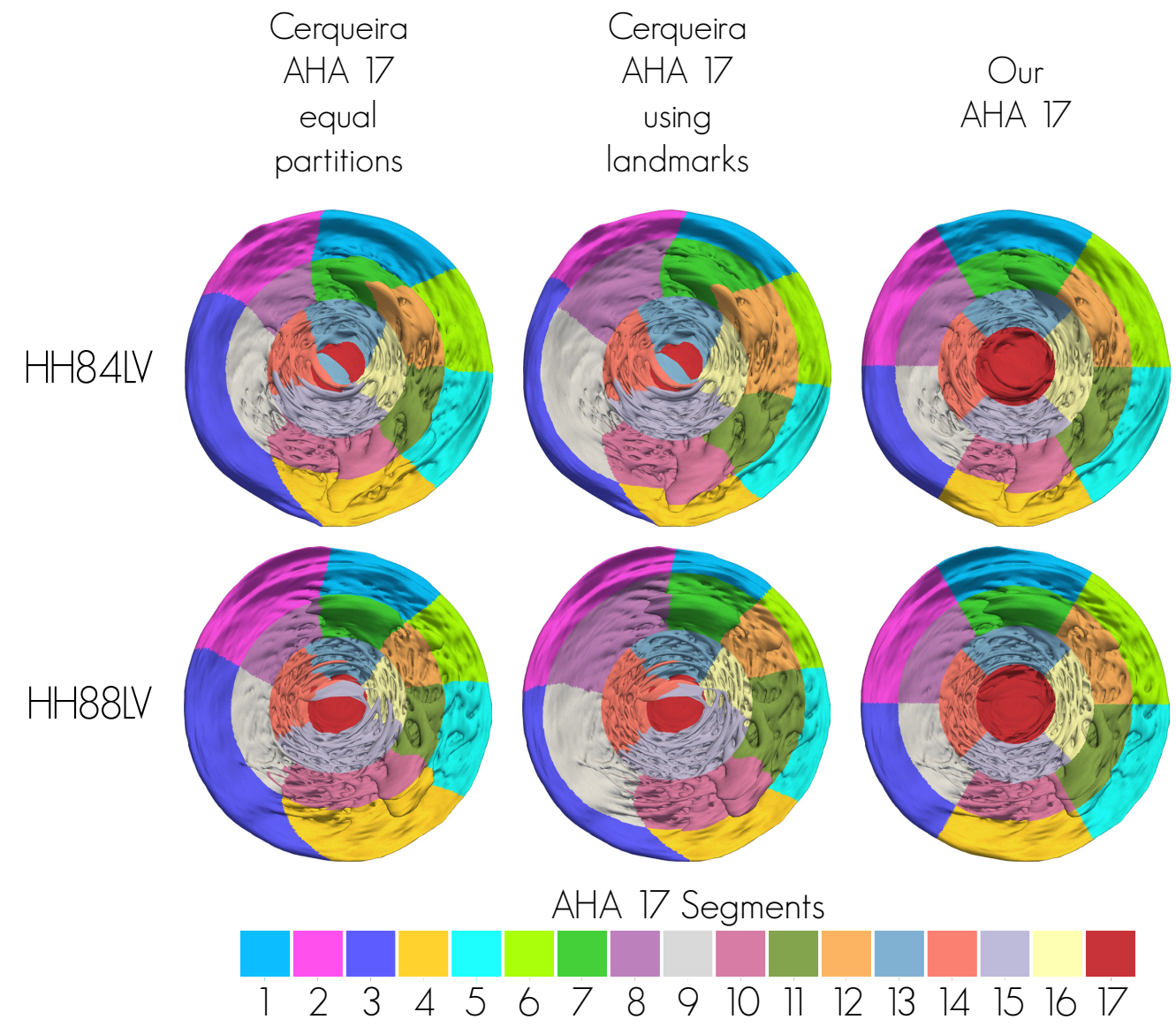

Figure 23: Partition of HH84 and HH88 LV meshes into 17 AHA represented in normalized reference frame.

If we follow additional guidelines proposed by Cerqueira et al. we can achieve more uniform segments as can be seen in the first and fourth row of Fig. 22. One can observe that partitioning by our method (Fig. 22, third and sixth row) produces similar results as obtained by the one proposed by Cerqueira et al. when the uniform partition is enforced.

Fig. 23 depicts $H H 84$ and $H H 88$ LVs AHA 17 segment information represented in our proposed reference frame. The first column represents the AHA segments obtained by Cerqueira method enforcing uniform partitions, the second column - AHA segments obtained by strictly following landmark 
locations proposed by Cerqueira et al., and the third column represents AHA segments defined by our method. We want to mention that with our method one can easily redefine the cardiac regions in any way by simply defining them on the disc, after that the regions can be easily mapped back onto any cardiac geometry. Furthermore, our method allows partitioning the ventricles in a transmural direction by labeling additional segments at different depths from the endocardium in the normalized representation.

\section{Discussion}

The proposed mapping allows us to define a patient independent reference frame, where populational statistical analysis can be performed or which can be used to map the detailed cardiac anatomy from one heart to another (represented by a smooth surface). To define the reference frame, we chose a cylindrical volumetric domain of unit height and a unit disk base for the $\mathrm{LV}$, while for the RV we chose a half cylinder domain of unit height and a half disk base as defined in section 2.3. The choice of the unit half disk and the unit disk as a the base parametric domain was motivated by the fact that such domains are already used by many authors for the visualization of any LV and RV information in the form of a bulls-eye plot (Cerqueira et al., 2002).

In relation to other approaches for ventricle re-parameterization, it is worth to note two general frameworks: use of prolate spheroidal coordinates and volumetric mesh fitting.

The prolate spheroidal coordinates such as used by Young et al. (2011) provide an interesting alternative to Cartesian coordinates as they allow to characterize any point on the elliptical surface in terms of 4 parameters: two of them related to the size of the ellipsoid and two angles (related to longitudinal and circumferential position of the point on the surface). Aligning this coordinate system with the cardiac left ventricle would allow representing every point on the cardiac surface using two parameters. However it should be noted that neither of cardiac ventricles is elliptical, especially the right ventricle, thus limiting the advantages of using the prolate spheroidal coordinates instead of Cartesian. On the other hand, just in our case, expressing cardiac data using prolate spheroidal coordinates would require aligning the

coordinate system with the ventricle by defining the long axis and some landmarks (for example, the centroids of valves). 
On the other hand the finite element models, such as used in Young et al. (2011); Gilbert et al. (2015), are more versatile in terms of parameterization and can be used to represent the whole of the cardiac chambers up to the valves with any number of holes. In this case, the point correspondence across the population is established using registration techniques or model fitting to an image. There are however certain drawbacks to using meshes for patient independent representation. The meshes based on Bézier and Hermite elements are limited to ventricles with smooth walls - having trabeculations adds topological variability to the mesh and hampers establishing point correspondence. On the other hand using tetrahedral or hexahedral meshes might be a better option for representing the meshes with varying topology, but again establishing correspondence in the face of varying topology is not trivial. It should be noted though that the mesh representations could be used to define a domain that encloses the trabeculations, reducing the problem of establishing correspondence to the one we have proposed in this paper.

We want to emphasize that our method is not limited to the mentioned planar domain shapes. The user can use a planar domain of any shape that is the most convenient or suits best for the job. In particular, in situations where one is not interested in visualization or analysis of the detailed cardiac anatomy, but solely in transferring such anatomy from one case to another, or to include it in a model which does not posses such structures, one can use the same, circular, planar domain for both ventricles.

The method proposed here is a generalization of our previously proposed parameterization method for the LV (Paun et al., 2015). It represents accurate and invertible mapping defined at every point inside both cardiac chambers and is not limited to any particular geometry of the chamber. Every point inside the cardiac chamber is characterized by 3 anatomically independent coordinates: two coordinates characterizing the point's projection onto the smooth anatomical bounding surface of the chamber and distance to that surface (longitudinal, circumferential positions and the depth).

The major challenge in the calculation of the coordinates is the calculation of the depth within myocardium, as depending on the shape of the cavity, trivial approaches like taking the distance along the normal will not work (this is especially true for the RV shapes). To deal with that challenge, the proposed method calculates a non-divergent vector field inside the chamber showing the flow from the bounding surface to the centroid of the mitral (tricuspid) annulus. This is achieved by solving the Laplace's equation in 
the interior of the chamber. The depth is then calculated from the distance along the corresponding streamline, thus providing an invertible mapping for any interior point independently of the geometry.

The main limitation of our method is the use of a discretized domain for the solution of the Laplace's equation. Normally, there is a high density of the streamlines close to the centroid of the mitral or tricuspid annulus (since this boundary is very small compared to the bounding surface). Given a fixed image and mesh resolutions in this neighborhood, whenever there are structures very close to the centroids, they tend to end up having distorted triangulation with self intersections. This problem however can be solved by increasing the resolution of meshes $M$ radially towards the centroid of the mitral or tricuspid annulus.

Another limitation is the manual landmark placement and the strategy of fixing the orientation in the proposed representation. From the experiments, one can see that the inaccuracy in landmark placement in the LV can lead to an angular error everywhere when different geometries are mapped onto our representation, while the errors are more localized in RV due to fixing the landmarks on our representation. We decided not to fix the two LV boundary landmarks on our representation to reduce possible distortion due to a more constrained mapping, however if these landmarks were fixed, just as in the case of RV, the inaccuracies in landmark placement would lead to much more localized errors. Furthermore, it is possible to automate the landmark placement using methods proposed by Karavides et al. (2010), Zheng et al. (2009a), Lu et al. (2009), Zheng et al. (2009b) or Lu et al. (2009).

The time and the accuracy of our algorithm depends on the element size of the discretized domain. The average total processing time of our algorithm executed on our 12 detailed anatomy meshes was 9 min $10 \mathrm{~s}$ on a single processor for the image size of $195 \times 228 \times 301$ voxels (isotropic voxel size of $0.3 \mathrm{~mm}$ ) and 3000 iterations. The average reconstruction error was on the level of $1 \mu \mathrm{m}$.

\section{Conclusion}

In this paper we presented a framework for mapping the meshes of right and left ventricular detailed cardiac anatomy to a common subject independent reference frame. The proposed method represents a major step towards the statistical analysis and quantification of different detailed ventricular 
morphologies among patients in a common framework with the potential application in computational cardiac models.

The framework allows mapping trabeculations from one heart geometry to another geometry given by a smooth surface. Thus, it is providing us the means of including different extracted trabecular morphologies to already available cardiac computational models, where the endocardial surfaces are modeled as smooth surfaces.

The code used in this manuscript as well as the segmentations of the detailed cardiac anatomy are publicly available and can be downloaded from http://physense.upf.edu. The original acquired datasets can be found and requested from http://www.vhlab.umn.edu/atlas/.

\section{Acknowledgments}

The Dicom datasets were provided by the Visible Heart ${ }^{\circledR}$ Laboratory (University of Minnesota), they were obtained by MRI scanning perfusion fixed hearts that were graciously donated by the organ donors and their families through LifeSource. B. Paun is supported by the grant FI-DGR 2014 (2014 FI B01238) from the Generalitat de Catalunya. The research leading to these results has received funding from the EU FP7 for research, technological development and demonstration under grant agreement VP2HF (no. 611823) and from the Spanish Ministry of Economy and Competitiveness (grant TIN2011-28067, TIN2014-52923-R, the Maria de Maeztu Units of Excellence Programme MDM-2015-0502) and FEDER.

\section{References}

Anderson, S.E., Hill, A.J., Iaizzo, P.A., 2009. Microanatomy of human left ventricular coronary veins. The Anatomical Record 292, 23-28.

Anderson, S.E., Quill, J.L., Iaizzo, P.A., 2008. Venous valves within left ventricular coronary veins. Journal of interventional cardiac electrophysiology 23, 95-99.

Attene, M., Falcidieno, B., 2006. Remesh: An interactive environment to edit and repair triangle meshes, in: Proc. Int. Conf. on Shape Modeling and Applications, pp. 41-41. 
Cerqueira, M.D., Weissman, N.J., Dilsizian, V., et al., 2002. Standardized myocardial segmentation and nomenclature for tomographic imaging of the heart a statement for healthcare professionals from the cardiac imaging committee of the council on clinical cardiology of the american heart association. Circulation 105, 539-542.

CIBC, 2015. Seg3D: Volumetric Image Segmentation and Visualization. Scientific Computing and Imaging Institute (SCI), Download from: http://www.seg3d.org [online: 10/08/2015].

De Craene, M., Tobon-Gomez, C., Butakoff, C., et al., 2012. Temporal diffeomorphic free form deformation (TDFFD) applied to motion and deformation quantification of tagged MRI sequences, in: Proc. Int. Conf. Statistical Atlases and Computational Models of the Heart. Imaging and Modelling Challenges, LNCS 7085, pp. 68-77.

Eggen, M.D., Swingen, C.M., Iaizzo, P.A., 2012. Ex vivo diffusion tensor MRI of human hearts: relative effects of specimen decomposition. Magnetic Resonance in Medicine 67, 1703-1709.

Floater, M.S., Hormann, K., 2005. Surface parameterization: a tutorial and survey, in: Advances in Multiresolution for Geometric Modelling, Mathematics and Visualization, pp. 157-186.

Gao, M., Chen, C., Zhang, S., et al., 2013. Segmenting the papillary muscles and the trabeculae from high resolution cardiac ct through restoration of topological handles, in: Proc. Int. Conf. Information Processing in Medical Imaging, LNCS 7917, pp. 184-195.

Gao, M., Chen, C., Zhang, S., et al., 2014. Morphological analysis of the papillary muscles and the trabeculae, in: Proc. Int. Sym. on Biomedical Imaging, pp. 373-376.

Garcia-Barnes, J., Gil, D., Badiella, L., et al., 2010. A normalized framework for the design of feature spaces assessing the left ventricular function. IEEE Trans. Medical Imaging 29, 733-745.

Gilbert, K., Lam, H.I., Pontré, B., Cowan, B., Occleshaw, C., Liu, J., Young, A., 2015. An interactive tool for rapid biventricular analysis of congenital heart disease. Clinical physiology and functional imaging . 
Goff, R.P., Spencer, J.H., Iaizzo, P.A., 2015. MRI reconstructions of human phrenic nerve anatomy and computational modeling of cryoballoon ablative therapy. Annals of biomedical engineering , 1-10.

Gu, X., Wang, Y., Chan, T.F., et al., 2004. Genus zero surface conformal mapping and its application to brain surface mapping. IEEE Trans. Medical Imaging 23, 949-958.

Haker, S., Angenent, S., Tannenbaum, A.R., et al., 2000. Conformal surface parameterization for texture mapping. IEEE Trans. Visualization and Computer Graphics 6, 181-189.

Hurdal, M.K., Stephenson, K., Bowers, P., et al., 2000. Coordinate systems for conformal cerebellar flat maps. NeuroImage 11, S467.

Joshi, A.A., Shattuck, D.W., Thompson, P.M., et al., 2007. Surfaceconstrained volumetric brain registration using harmonic mappings. IEEE Trans. Medical Imaging 26, 1657-1669.

Karavides, T., Leung, K.E., Paclik, P., Hendriks, E.A., Bosch, J.G., 2010. Database guided detection of anatomical landmark points in 3D images of the heart, in: SPIE Medical Imaging, pp. 76234Q-76234Q.

Karim, R., Ma, Y., Jang, M., et al., 2014. Surface flattening of the human left atrium and proof-of-concept clinical applications. Computerized Medical Imaging and Graphics 38, 251-266.

Kharevych, L., Springborn, B., Schröder, P., 2006. Discrete conformal mappings via circle patterns. ACM Trans. Graphics 25, 412-438.

Lorensen, W.E., Cline, H.E., 1987. Marching cubes: A high resolution 3D surface construction algorithm, in: Proc. Conf. Computer Graphics and Interactive Techniques, pp. 163-169.

Lu, X., Georgescu, B., Littmann, A., Mueller, E., Comaniciu, D., 2009. Discriminative joint context for automatic landmark set detection from a single cardiac MR long axis slice, in: Int. Conf. Functional Imaging and Modeling of the Heart, LNCS 5528, pp. 457-465.

Paun, B., Bijnens, B., Butakoff, C., 2015. Subject independent reference frame for the left ventricular detailed cardiac anatomy, in: Int. Conf. Functional Imaging and Modeling of the Heart, LNCS 9126, pp. 240-247. 
Pinkall, U., Polthier, K., 1993. Computing discrete minimal surfaces and their conjugates. Experimental mathematics 2, 15-36.

Riemann, B., Wilkins, D.R., 1851. Grundlagen für eine allgemeine Theorie der Functionen einer veränderlichen complexen Grösse. Ph.D. thesis. EA Huth.

Schroeder, W.M., Martin, K., Lorensen, B., 2006. The Visualization Toolkit: An Object Oriented Approach to 3D Graphics 3rd Edition. Kitware Inc.

Sheffer, A., Praun, E., Rose, K., 2006. Mesh parameterization methods and their applications. Foundations and Trends in Computer Graphics and Vision 2, 105-171.

Soto-Iglesias, D., Butakoff, C., Andreu, D., et al., 2013. Evaluation of different mapping techniques for the integration of electro-anatomical voltage and imaging data of the left ventricle, in: Proc. Int. Conf. Functional Imaging and Modeling of the Heart, LNCS 7945, pp. 391-399.

Squillacote, A.H., 2008. The ParaView Guide: A Parallel Visualization Application. 3rd ed., Kitware Inc.

Tobon-Gomez, C., Zuluaga, M.A., Chubb, H., et al., 2015. Standardised unfold map of the left atrium: regional definition for multimodal image analysis. Journal of Cardiovascular Magnetic Resonance 17, 1-3.

Tutte, W.T., 1963. How to draw a graph. Proc. London Mathematical Society 13, 743-768.

Vera, S., González-Ballester, M.A., Gil, D., 2014. Anatomical parameterization for volumetric meshing of the liver, in: Proc. SPIE, pp. 903605903605.

Wang, Y., Shi, J., Yin, X., et al., 2012. Brain surface conformal parameterization with the ricci flow. IEEE Trans. Medical Imaging 31, 251-264.

Yezzi, A.J., Prince, J.L., 2003. An eulerian PDE approach for computing tissue thickness. IEEE Trans. Medical Imaging 22, 1332-1339.

Young, A.A., Crossman, D.J., Ruygrok, P.N., Cannell, M.B., 2011. Mapping system for coregistration of cardiac MRI and ex vivo tissue sampling. Journal of Magnetic Resonance Imaging 34, 1065-1071. 
Young, A.A., French, B.A., Yang, Z., Cowan, B.R., Gilson, W.D., Berr, S.S., Kramer, C.M., Epstein, F.H., 2006. Reperfused myocardial infarction in mice: 3D mapping of late gadolinium enhancement and strain. Journal of Cardiovascular Magnetic Resonance 8, 685-692.

Zhang, X., Cowan, B.R., Bluemke, D.A., Finn, J.P., Fonseca, C.G., Kadish, A.H., Lee, D.C., Lima, J.A., Suinesiaputra, A., Young, A.A., et al., 2014. Atlas-based quantification of cardiac remodeling due to myocardial infarction. PLoS ONE 9, e110243.

Zheng, Y., Georgescu, B., Comaniciu, D., 2009a. Marginal space learning for efficient detection of 2D/3D anatomical structures in medical images, in: Information Processing in Medical Imaging, pp. 411-422.

Zheng, Y., Georgescu, B., Ling, H., Zhou, S.K., Scheuering, M., Comaniciu, D., 2009b. Constrained marginal space learning for efficient 3D anatomical structure detection in medical images, in: IEEE Conference on Computer Vision and Pattern Recognition, pp. 194-201. 